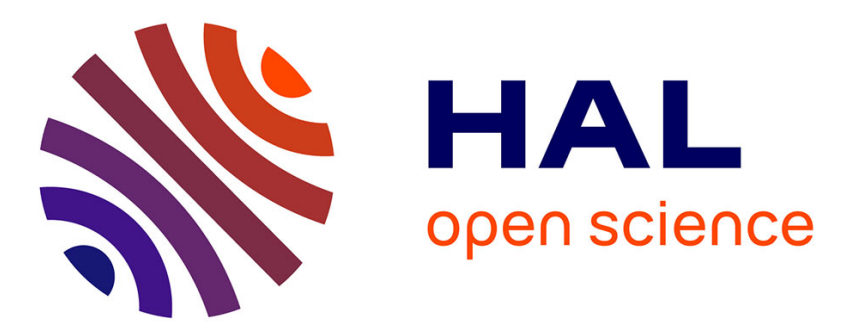

\title{
TWIG: A model to simulate the gravitropic response of a tree axis in the frame of elasticity and viscoelasticity, at intra-annual time scale
}

Coutand Catherine, Mathias Jean-Denis, Jeronimidis Georges, Destrebecq Jean-François

\section{To cite this version:}

Coutand Catherine, Mathias Jean-Denis, Jeronimidis Georges, Destrebecq Jean-François. TWIG: A model to simulate the gravitropic response of a tree axis in the frame of elasticity and viscoelasticity, at intra-annual time scale. Journal of Theoretical Biology, 2011, 273 (1), pp.115. 10.1016/j.jtbi.2010.12.027 . hal-00669203

\section{HAL Id: hal-00669203 https://hal.science/hal-00669203}

Submitted on 12 Feb 2012

HAL is a multi-disciplinary open access archive for the deposit and dissemination of scientific research documents, whether they are published or not. The documents may come from teaching and research institutions in France or abroad, or from public or private research centers.
L'archive ouverte pluridisciplinaire HAL, est destinée au dépôt et à la diffusion de documents scientifiques de niveau recherche, publiés ou non, émanant des établissements d'enseignement et de recherche français ou étrangers, des laboratoires publics ou privés. 


\section{Author's Accepted Manuscript}

TWIG: A model to simulate the gravitropic response of a tree axis in the frame of elasticity and viscoelasticity, at intra-annual time scale

Coutand Catherine, Mathias Jean-Denis, Jeronimidis Georges, Destrebecq Jean-François

$\begin{array}{ll}\text { PII: } & \text { S0022-5193(10)00682-X } \\ \text { DOI: } & \text { doi:10.1016/j.jtbi.2010.12.027 } \\ \text { Reference: } & \text { YJTBI6296 }\end{array}$

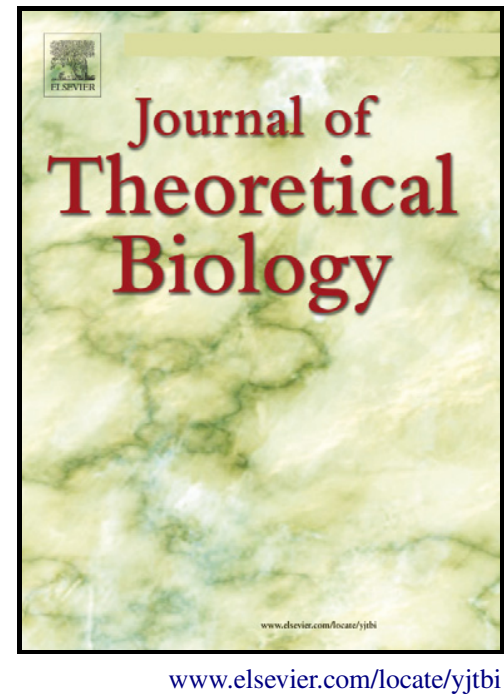

To appear in: $\quad$ Journal of Theoretical Biology

Received date: 9 July 2010

Revised date: $\quad 8$ November 2010

Accepted date: 17 December 2010

Cite this article as: Coutand Catherine, Mathias Jean-Denis, Jeronimidis Georges and Destrebecq Jean-François, TWIG: A model to simulate the gravitropic response of a tree axis in the frame of elasticity and viscoelasticity, at intra-annual time scale, Journal of Theoretical Biology, doi:10.1016/j.jtbi.2010.12.027

This is a PDF file of an unedited manuscript that has been accepted for publication. As a service to our customers we are providing this early version of the manuscript. The manuscript will undergo copyediting, typesetting, and review of the resulting galley proof before it is published in its final citable form. Please note that during the production process errors may be discovered which could affect the content, and all legal disclaimers that apply to the journal pertain. 
TWIG: a model to simulate the gravitropic response of a tree axis in the frame of elasticity and viscoelasticity, at intra-annual time scale.

\section{Coutand Catherine ${ }^{a^{*}}$, Mathias Jean-Denis ${ }^{b^{*}}$, Jeronimidis Georges ${ }^{c^{*}}$, Destrebecq Jean- François ${ }^{*}$}

${ }^{\text {a } I N R A ~ / ~ u m r ~} 547$ PIAF (Physique et physiologie Intégratives de l'Arbre Fruitier et forestier), 234 avenue du Brézet, F-63000Clermont-Ferrand, France, coutand@clermont.inra.fr

${ }^{\mathrm{b}}$ Laboratoire d'Ingénierie pour les Systèmes Complexes, CEMAGREF, Campus des Cézeaux, 24 avenue des Landais - BP 50085, 63172 Aubière Cedex, France, jeandenis.mathias@cemagref.fr

${ }^{\mathrm{c}}$ School of Construction Management and Engineering, University of Reading, Whiteknights, Reading RG6 2AY, United Kingdom, G.Jeronimidis@reading.ac.uk

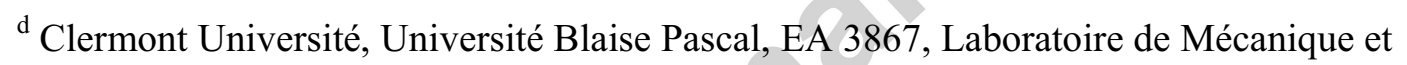
Ingénieries (LaMI), BP 10448, F-63000 Clermont-Ferrand, France, jeanfrancois.destrebecq@,univ-bpclermont.fr

* The authors contributed equally to this work.

Corresponding author:

Catherine Coutand

Postal address: Umr 547 PIAF, INRA, 234 avenue du Brézet, F-63100 Clermont-Ferrand Mail: coutand@,clermont.inra.fr

Tel: +33473624673

Fax: +33473624454 


\section{Abstract}

Trees are able to maintain or to modify the orientation of their axes (trunks or branches) by tropic movements. For axes in which elongation is achieved but cambial growth active, the tropic movements are due to the production of a particular wood, called reaction wood which is prestressed within the growing tree. Several models have been developed to simulate the gravitropic response of axes in trees due to the formation of reaction wood, all within the frame of linear elasticity and considering the wood maturation as instantaneous. The effect viscoelasticity of wood has, to our knowledge, never been considered. The TWIG model presented in this paper aims at simulating the gravitropic movement of a tree axis at the intraannual scale. In this work we studied both the effect of a non instantaneous maturation process and of viscoelasticity. For this purpose, we considered the elastic case with maturation considered as an instantaneous process as the reference. The introduction of viscoelasticity in TWIG has been done by coupling TWIG to a model developed for bridges. Indeed from a purely mechanical point of view, bridges and trees are very similar: they are structures which are built in stages, they are made of several materials (composite structures), their materials are prestressed (wood is prestressed during the maturation process as a result of polymerisation of lignin and cellulose to form the secondary cell wall and concrete is prestressed during drying). Simulations gave evidence that the reorientation process of axes can be significantly influenced by the kinetics of maturation. Moreover the model has now to be tested with more experimental data of wood viscoelasticity but it appears that in the range of a relaxation time from 0 to 50 days, viscoelasticity has an important effect on the evolution of tree shape as well as on the values of prestresses.

Key words: biomechanics, modelling, tree, viscoelasticity, gravitropism 


\section{Introduction}

\subsection{Tree growth}

Tree growth is ensured by the activity of growing zones with meristems. Meristematic activity in buds and expanding zone provide the elongation growth of axes. Activity of the cambium ensures diameter growth of trunk and branches. Cambium produces new cells by divisions. These cells first enlarge in (diameter) and then enter a phase of maturation i.e. a phase where cellulose and lignins are polymerized to constitute the secondary cell wall that provides wood rigidity. Stresses are generated during this maturation phase [1-3]. Little is known about this maturation phase in terms of kinetics. A few old studies give the maturation time of a cell about 1 to 2 weeks $[4,5]$. From a mechanical point of view, growth in diameter can be seen as successive depositions of wood layers on an existing internally stressed structure.

\subsection{Gravitropism in trees}

A tree has to develop its axes in order to capture light and to carry its own weight without buckling. Tree axes are not passive structures; gravitropism and phototropism are ways for trees to control their posture by maintaining and modifying the orientation of trunk and branches in space $[6,7]$. In elongating organs (for trees, annual shoots at the beginning of the growing season), the gravitropic movement is ensured by differential growth (one side of the organ elongates more rapidly than the other); in organs where elongation is achieved but radial growth is active, the gravitropic movement is due to the production of reaction wood [8] on one side of the axis. The differentiation of reaction wood is often accompanied by an eccentricity of diameter growth. The gravitropic response of plants has been mainly studied on young elongating organs such as coleoptiles, hypocotyles or epicotyles. The description of the kinematics of the gravitropic response on radially growing organs like tree trunks (except the annual shoot) are sparse, except some studies on woody gymnosperms [9-11] and two recent studies of the gravitropic response of artificially tilted woody angiosperms $[12,13]$. An 
example of the successive shapes of a poplar trunk is shown on Figure 1. The tropic response consists of three phases. During phase 1, one or two weeks after tilting, the shape of the trunk remains unchanged or the trunk even sags (latency phase). In fact, differentiation of tension wood fibers in artificially tilted poplar has been shown to occur about 48 hours after tilting [14]. The latency phase is thus probably due to the maturation phase of tension wood. Phase 2 corresponds to the upward curvature of the trunk two or three weeks after tilting. In phase 3, about four to five weeks after tilting, a local decurving process takes place, enabling the trunk to straighten back to vertical. This decurving process starts at the top of the trunk and propagates towards the trunk base [12]. The same trends can be inferred from the data shown in [11] in the case of a conifer.

\subsection{Modelling the gravitropic response of a tree axis}

Several publications have been dedicated to model the gravitropic response of axes in trees due to radial growth and the production of reaction wood [11, 15-19]. All these models were developed within the frame of linear elasticity.

The model of Fournier predicts that the variation of curvature of a given cross section due to the formation of reaction wood is proportional to half the difference of maturation strains between normal and reaction wood $(\Delta \beta)$, as shown in Equation (1), see [11] for more details:

$$
d \chi=4 \Delta \beta \frac{d R}{R^{2}}
$$

where $\mathrm{d} \chi$ is the change of curvature, $\mathrm{dR}$ the radial growth and $\mathrm{R}$ the radius.

The simulation of the successive shapes of the trunk requires a law to predict the location and the extent of reaction wood sectors or direct measurements of these parameters. In addition, a regulation hypothesis is needed to relate the formation of tension wood to the local inclination of a trunk sector for angles between 0 and 90 to the horizontal.

The Fournier model assumes also that the maturation time of the wood, i.e. the time during which the maturation stresses appear in wood, is negligible with respect to the diameter 
growth rate of the tree. This is equivalent to assuming that $\mathrm{d} \chi$ occurs at the same rate as $\mathrm{dR}$ and that time, as a variable, is not explicitly included in the model. As stated in Fournier et al. 1994, the model simulates nicely the successive shapes of the trunk of a young Pinus pinaster artificially tilted but, as stated in [20], the value of the difference of maturations strains used in the simulations was very high $(0.25 \%)$.

Assuming $\Delta \beta$ constant and integrating both sides of Equation (1) a plot of $\Delta \chi$ versus $\left(1 / R_{i}\right.$ $1 / \mathrm{R}_{\mathrm{f}}$ ), where $\Delta \mathrm{R}=\mathrm{R}_{\mathrm{i}}-\mathrm{R}_{\mathrm{f}}$, should give a straight line with slope $\Delta \beta$ and zero intercept. When compared with experimental results obtained with young woody angiosperms $[12,13]$, the correlation between $\Delta \chi$ and $\left(1 / \mathrm{R}_{\mathrm{i}}-1 / \mathrm{R}_{\mathrm{f}}\right)$ was found to be weak, the slope suggesting a much greater value of $\Delta \beta$, compared to measured data and a non-zero intercept. In this comparison, the assumption of an instantaneous wood maturation is equivalent to taking $\Delta \chi$ and $\left(1 / R_{i}\right.$ $1 / R_{f}$ ) at the same time step. Considering the measured values of the rates of change with time of curvatures and radial growth increments, a phase difference in time between the two is observed. This phase difference may be due to the maturation process which is not instantaneous and to the difference of maturation strains which is not constant. In [12] the main reasons invoked to explain these discrepancies between the model and the experimental data were: i) spatio-temporal variations of the maturation strains in wood, ii) maturation time not negligible compared to diameter growth rate when the time step is of the order of a week. For a given time step the active wood annulus that leads to curvature is not the annulus of diameter growth measured during that time step but the wood annuli produced during preceding time steps. This important feature has, to our knowledge, never been evoked in previous work on modelling tree axes reorientations.

Up to now the models dedicated to simulate the gravitropic reaction of trees have been developed in the frame of linear elasticity. As shown in this study, the elastic model does not agree with measured values. Values of $\Delta \beta$ are not known with high accuracy and it is always 
possible to use values which will make the results of simulations (curvatures) agree with measured values. However, as shown in this paper, including the viscoelastic behaviour of wood in the model results in a better agreement with the experimental values even with using measured values of $\Delta \beta$. In addition, wood, and especially green wood, is known to be a viscoelastic material [21-23], i.e. its rheological behaviour depends on time and is relevant to the gravitropic response of trees.

\subsection{Mechanical models in engineering}

Earlier models of tree gravitropic reactions have been developed using elastic properties of materials. Recently, researchers from the mechanical engineering community [24-26] have developed a model able to simulate the evolution of the stresses with time within a structure consisting of several viscoelastic materials and built in stages. Their model was developed in the frame of linear viscoelasticity.

From a mechanical point of view, models developed for tree gravitropic reactions and those developed for bridges present several similarities. Concrete bridges and trees are made of several materials; they are heterogeneous composite structures and they are built up in phases and their materials are prestressed. Wood is prestressed during the maturation process (polymerisation of lignin and cellulose to form the secondary cell wall); concrete bridges are mechanically prestressed to improve their load-bearing capacity.

In the present paper the models have been combined to develop a more general model (TWIG) able to simulate the successive shapes and the evolution of the internal mechanical states of a trunk as a "growing" beam with circular cross section.

The first part of the paper recalls the features of tree growth and wood maturation relevant to modelling. The second describes the mechanical problem associated with a tree's reorientating axis and presents the incremental formulation for the numerical modelling of viscoelastic materials and the way such a model has been implemented in TWIG. In the third 
part of the paper, the model is used to simulate the reorientation progress of a tree axis. Simulations were first done in the frame of linear elasticity. The time step is the week, a time step for which the kinetics of maturation might not be negligible regarding the diameter growth rate. We thus tested two models of kinetics of maturation. A study of sensitivity to the values of the parameters is presented. Later simulations were done in the frame of linear viscoelasticity in order to estimate the consequences of wood viscoelasticity on the rightingup process and are presented in the fourth part of the paper.

\section{Mechanical modelling}

\subsection{Description of the mechanical problem of a reorienting axis}

A tree axis can be considered in the frame of beam theory but it is not a classic problem because the beam is 'growing'. As growth can be seen as successive depositions of additional material, mass and geometry change with time in a growing structure [11]. The mechanical problem in the case of the reorientation of a growing axis can be considered as that of a beam loaded by two types of forces: the self-weight of the axis and the action of asymmetrical internal maturation stresses due to the development of a sector of reaction wood (Figure 2). However, because of the on-going changes in the mass and geometry of the growing axis, the beam problem must be solved using an incremental approach where the parameters vary as a function of time.

When wood is not considered as an elastic material but as a viscoelastic one, an incremental approach is also required in order to simulate the evolution of the axis shape and of the prestresses with time. Before describing the calculations of the model, including the incremental formulation due to the growth of tree axis and the implementation of viscoelasticity into the model, we will briefly remind the basic aspects of viscoelasticity relevant to this work. 


\subsection{Constitutive law of a viscoelastic material}

When a constant state of stress is applied to a viscoelastic material (e.g. wood), creep is observed in the course of time: the strain is progressively increasing until it reaches a steady value (Figure 3B). As a corollary, if a viscoelastic material is subjected to a constant state of strain, a progressive relaxation of the induced stress is observed in the course of time (Figure 3A). These two phenomena describe the time dependent behaviour of the material which may be analyzed in the frame of the theory of linear viscoelasticity [27-30]. According to this theory, the stress $\sigma(t)$ resulting at any time $t>0$ from an imposed history of strain $\varepsilon(t)$ is given by Boltzmann's equation [29]:

$$
\sigma(\mathrm{t})=\int_{0}^{t} R(\mathrm{t}, \tau) \dot{\varepsilon}(\tau) d \tau+\sum_{i} H\left(t-t_{i}\right) R\left(t, t_{i}\right) \Delta \varepsilon\left(t_{i}\right)
$$

where $\dot{\varepsilon}(t)$ is the time derivative of the strain loading function; $\Delta \varepsilon\left(t_{i}\right)$ denotes a set of strain discontinuities occurring at specified times $t_{i}$ and $H(t)$ is the Heavyside function.

In this fundamental expression, $R\left(t, t_{\mathrm{i}}\right)$ is a relaxation function which depends on the mechanical properties of the material and on the period of time $t$ - $t_{\mathrm{i}}$ elapsed since the application of a constant imposed strain. Owing to the principles of thermodynamics applied to the mechanics of viscoelastic media [30], this function can be developed as a sum of decaying exponential terms in the form of a Dirichlet's series with $t_{0}$ the time at which a constant strain is imposed:

$$
R\left(t, t_{0}\right)=\sum_{i=0}^{r} E_{i} e^{-\alpha_{i}\left(t-t_{o}\right)}
$$

where $E_{\mathrm{i}}$ and $\alpha_{\mathrm{i}}$ are material parameters. This relationship can be represented by an analogous generalized Maxwell's chain with $r+1$ branches (see Fig. 4A), where branch 0 consists of a spring (elastic), and each other branch consists of a spring (elastic) connected to 
a dashpot (viscous). $\alpha_{\mathrm{i}}^{-1}=\eta_{\mathrm{i}} / E_{\mathrm{i}}$ is called relaxation time of branch $i$ (with $\alpha_{0}=0$ ). For $r=1$, the generalized Maxwell's chain is known as Zener's model (see Fig. 4B). As already mentioned in previous sections, a growing stem exhibits a heterogeneous cross-section constituted of two types of wood, namely normal wood and reaction wood (see Fig. 2). In the following, both types of wood will be considered as viscoelastic. Hence, Eq. 3 will be used to model the mechanical behaviour of each type of wood in the course of time.

\subsection{Incremental formulation of the constitutive law}

Various methods may be used to solve viscoelastic problems. Most of them require a large memory size and heavy processing time. In contrast, the method used in this paper requires limited memory size and short computation time. It is based on an incremental formulation of the viscoelastic constitutive law, which has been initially set for concrete material [31]. Later on, the method has been improved and adapted to the analysis of composite structures made of several elastic or viscoelastic materials [26]. This method has proved to be quite efficient in the analysis of time dependent effects in complex structures such as huge concrete bridges $[24]$.

The method is based on the integration of Eq. (2) over a finite time interval $[t, t+\Delta t]$, the relaxation function being expressed as a Dirichlet's series (Eq. (3)). Hence,

$\forall t, \Delta t: \quad \Delta \sigma=\sum_{i=0}^{r} E_{i}\left(\int_{0}^{t+\Delta t} e^{-\alpha_{i}(t+\Delta t-\tau)} \dot{\varepsilon}(\tau) d \tau-\int_{0}^{t} e^{-\alpha_{i}(t-\tau)} \dot{\varepsilon}(\tau) d \tau\right)$

Under the assumption that the strain rate $\dot{\varepsilon}(t) \approx \Delta \varepsilon / \Delta t$ is about constant over the finite time interval $\Delta t$, this equation yields:

$\forall t, \Delta t: \quad \Delta \sigma=E^{*}\left(\Delta \varepsilon-\Delta \varepsilon^{*}\right)+\sigma^{h i s}(t)$

where

$E^{*}=\sum_{i=0}^{r} E_{i}^{*}=E_{0}+\frac{1}{\Delta t} \sum_{i=1}^{r} \frac{E_{i}}{\alpha_{i}}\left(1-e^{-\alpha_{i} \Delta t}\right)$ 


$$
\sigma^{h i s}(t)=\sum_{i=0}^{r} \sigma_{i}^{h i s}(t)=-\sum_{i=0}^{r}\left(1-e^{-\alpha_{i} \Delta t}\right) \sigma_{i}(t)
$$

Equation (5) is the constitutive law of a viscoelastic material expressed in an incremental form. In this equation, $E^{*}$ plays the role of a fictitious modulus of elasticity whose actual value depends on the material parameters $\alpha_{\mathrm{i}}$ and $E_{\mathrm{i}}$, and on the length of the time interval $\Delta t$. $\Delta \varepsilon^{*}$ is a free-strain increase (or decrease) occurring during the time interval $\Delta t . \sigma^{h i s}(t)$ is a term which summarises the effects of the total stress history since the beginning of the loading period. $\sigma_{i}(t)$ is an internal stress attached to branch $i$ of the generalized Maxwell's chain; its value must be updated after each time interval, using Eq. (5):

$\forall t, \Delta t: \quad \sigma_{i}(\mathrm{t}+\Delta \mathrm{t})=\sigma_{i}(\mathrm{t})+E_{i}^{*}\left(\Delta \varepsilon-\Delta \varepsilon^{*}\right)+\sigma_{i}^{h i s}(t)$

Further details may be found in [24, 26].

\subsection{Constitutive equation for a viscoelastic beam}

In the following, [] is used for square matrices, \{\} or $<>$ are used for vectors (column or row matrices respectively). The kinematic equations of a heterogeneous beam cross-section made of $n$ homogeneous viscoelastic subsections (see Figure 5) and subjected to in-plane loading, may be written as follows in an incremental form:

$\forall t, \Delta t: \quad\{\Delta F\}=\sum_{j=1}^{n}\left\{\Delta F_{j}\right\}$ where $\quad\left\{\Delta F_{j}\right\}=\int_{A_{j}}\left\{\begin{array}{l}1 \\ y\end{array}\right\} \Delta \sigma d A$

where $A_{j}$ is the cross-area of subsection $j, y$ is the distance to the reference $x$-axis of the beam cross-section, $\Delta \sigma$ is given by Eq. (5) and $\{\Delta F\}$ is defined below (see Eq. (12)).

On account of Bernoulli's principle applied to the cross-section as a whole, the increment in the longitudinal strain at any location within the beam cross-section is given by:

$$
\forall t, \Delta t: \quad \Delta \varepsilon=\langle 1 \quad y\rangle\{\Delta D\}
$$

where $\langle 1 \quad y\rangle$ is the transpose matrix of $\left\{\begin{array}{l}1 \\ y\end{array}\right\}$ and $\{\Delta D\}$ is defined below (see Eq. (12)). 
Taking Eqs. (5) and (10) into account, Eq. (9) may be easily integrated. Details about the procedure of integration may be found in [26]. It yields:

$$
\forall t, \Delta t: \quad\{\Delta F\}=\sum_{j=1}^{n}\left\{\Delta F_{j}\right\}=[\Pi]\{\Delta D\}+\left\{F^{h i s}\right\}-\left\{\Delta F^{*}\right\}
$$

This incremental formulation is the constitutive equation of the heterogeneous viscoelastic beam cross-section. The meanings of the different terms in this equation are given below:

- $\{\Delta F\}$ and $\{\Delta D\}$ represent the increments in the generalized stresses and strains in the beam cross-section over the given time interval:

$$
\{\Delta F\}=\left\{\begin{array}{c}
\Delta N_{z} \\
\Delta M_{x}
\end{array}\right\} \text { and }\{\Delta D\}=\left\{\begin{array}{c}
\Delta \varepsilon_{0} \\
\Delta \chi
\end{array}\right\}
$$

$\Delta N_{x}$ and $\Delta M_{y}$ are the increments of normal force and bending moment, respectively; $\Delta \varepsilon_{0}$ and $\Delta \chi$ are the increments of longitudinal strain and curvature, respectively.

- $\left\{F^{h i s}\right\}$ and $\left\{\Delta F^{*}\right\}$ are two loading vectors that allow for the stress history since the beginning of loading and for free-strain increment during the time interval, respectively:

$$
\begin{aligned}
& \left\{F^{\text {his }}\right\}=\left\{\begin{array}{l}
N_{z}^{\text {his }} \\
M_{x}^{\text {his }}
\end{array}\right\}=\sum_{j=1}^{n} \int_{A_{j}}\left\{\begin{array}{l}
1 \\
y
\end{array}\right\} \sigma_{j}^{\text {his }}(t) d A \\
& \left\{\Delta F^{*}\right\}=\left\{\begin{array}{c}
\Delta N_{z}^{*} \\
\Delta M_{x}^{*}
\end{array}\right\}=\sum_{j=1}^{n} \int_{A_{j}}\left\{\begin{array}{l}
1 \\
y
\end{array}\right\} E_{j}^{*} \Delta \varepsilon_{j}^{*} d A
\end{aligned}
$$

- $[\Pi]$ is a fictitious stiffness matrix for the heterogeneous cross-section, calculated with the fictitious modulus of elasticity (see Eq. (6)):

$$
[\Pi]=\sum_{j=1}^{n} E_{j}^{*}\left[K_{j}\right] \text { where } \quad\left[K_{j}\right]=\left[\begin{array}{cc}
A_{j} & P_{j} \\
P_{j} & I_{j}
\end{array}\right]
$$

where $A_{j}$ is the cross-sectional area, $P_{j}$ and $I_{j}$ are the first and the second moments of inertia of subsection $j$, respectively. 
Using Eq. (11) the problem is turned into an incremental procedure where every step consists in the resolution of a fictitious elastic problem. The only approximation made is that the strain rate is approximately constant over the time interval $\Delta t$, which therefore needs to be finite but not necessarily short. As a matter of fact, its length can be adjusted according to the strain rate evolution during the analysis.

For the sake of simplicity the equations describing eccentricity law, weight increment, wood maturation process, trunk diameter evolution, etc., are not included here. The details of the model are available in Appendix A.

\section{Application to a growing tree}

In this part we discuss the data used for simulations. We first tested the influence of parameter values on simulations when considering wood as an elastic material; then we considered wood as a viscoelastic material and checked the influence of the viscoelastic parameters values. Matlab (7.2) for Windows (The MathWorks@) was used for the whole programming of the current model. In the following, only the initial weight of the stem and the additional weight of the successive growth layers are taken into account. The weight of shoots and leaves is neglected; this assumption is consistent with the fact that the annual and lateral shoots and the leave weight were limited on the young poplars from which the experimental data were derived for the present study. This assumption does not change the principles of the analysis, although it may slightly affect the results from a quantitative standpoint. The model has been validated using simple cases. The validation results are available in Appendix B.

\subsection{Data used for simulations}

The knowledge of several quantities is required in order to adapt the model described above to the analysis of the reorientation process of a tree axis during a period of growth. Appropriate assumptions are based on experimental evidences [12]: 
- during a period of growth, the longitudinal profile of a trunk evolves due to the progressive deposition and setting of thin additional wood layers. As mentioned above, the axial growth is not considered in the following (weight of the annual shoot neglected);

- the evolution of the cross-section diameter along the trunk follows a quadratic function based on experimental data:

$$
\begin{array}{ll}
\text { initial : } & R_{i n i}=-0.0011 x^{2}-0.0014 x+0.009 \\
\text { final : } & R_{i n i}=0.0011 x^{2}-0.00589 x+0.01349
\end{array}
$$

where $x$ denotes the location along the geometrical line of the trunk. $R_{i n i}$ and $R_{f i n}$ are functions which enable to compute the values of the stem radius along the trunk at the beginning and the end of the growing period, respectively. In the present simulation, tree cambial growth is assumed to be linear in the course of time, i.e. the trunk diameter is increasing at a constant rate but non-linear cambial growth rate can be also used in the model;

- the cross-section of a trunk grows in an asymmetrical manner during a process of reorientation (Figure 5). The radius of the cross-section is progressively increased from its initial value $R_{i n i}$ to its final value $R_{\text {fin }}$. Each new layer is made of normal wood (NW) and of a sector of tension wood (TW) defined by a sector-angle ( $\theta$ ) (Figure 5). The asymmetry in the radial growth can be taken into account by shifting the centre of every new growth ring by a distance $(\delta)$ measured from the centroid of the initial crosssection. The relationship between the distance $(\delta)$ and the actual radius of the growing axis is given in Appendix A.3; 
- a significant shrinkage occurs in every new layer after its maturation; combined with the asymmetrical growth mentioned above, this phenomenon enables the process of reorientation of the trunk;

- tension wood exhibits higher rigidity than normal wood, as verified experimentally [32].

A Zener model (Figure 4B) is used to represent the viscoelastic behaviour of each kind of wood which, as a special case, can also describe the elastic behaviour.

The trunk of the tree is considered as a growing structure made of an initial core of normal wood and a progressive addition of thin heterogeneous layers made of normal and tension wood according to Figure 5. For the analysis, the trunk is divided in short heterogeneous beam segments, and the period of growth is divided in elementary steps. The cross-sectional characteristics of each segment are considered as constant during every growth and viscoelastic step.

Due to the process of reorientation, the geometry of the trunk evolves in a significant manner. This evolution is precisely taken into account in the calculation process: the geometry is updated for each step of growth and each viscoelastic step. Two sets of reference axes are used to this purpose: the incremental resolution of Eq. (11) is performed for every step of growth in the local reference axes attached to each beam segment, whereas general reference axes are used to modify the coordinates of each segment i.e. to update the geometry of the trunk after every calculation step.

Each elementary growth step corresponds to the setting of a new heterogeneous layer. Shrinkage is assumed to occur in a new layer at the time of setting, i.e. at the beginning of the current step. This is taken into account in the model through the "free strain" increase $\Delta \varepsilon^{*}$ (see Eqs.(5) and (8) and (14)). At the same time, the mass and rigidity of the new layer are added to the existing structure. These mass and rigidity are considered as constant at each step 
of time. These phenomena cause an evolution in the internal forces as well as in the strains and the displacements due to the elastic reactions of the different parts of the structure.

When considering woods (normal wood and tension wood) as viscoelastic materials, an iterative procedure is used for the analysis of the heterogeneous viscoelastic structure in the course of time. For this purpose, each elementary growth step is divided into short time intervals. A creep calculation based on Equation (11) is performed for each time interval. It yields an increase in the strains and the displacements as well as a change in the internal forces of the structure due to the rheological behaviour and the heterogeneous nature of the cross-sections. After every calculation step the stresses are calculated and updated throughout every cross-section and the geometry of every segment is updated. The flowchart shown in Figure 6 summarizes the main features of the calculation process.

Simulations done to check the influence of the number of viscoelastic steps on the computed curvature show that the number of steps should be at least 20 to get a steady response (Figure 7). This value is thus retained in the further simulations.

The regulation law used for the control of reaction wood formation is binary, similar to the one described in Fournier's model [11] but adapted for deciduous trees: for each tilted trunk segment, tension wood forms on the upper part of the segment; the formation is stopped when the segment reaches the vertical direction (in this case, normal wood only is produced); tension wood forms on the opposite side of the trunk if it overshoots the vertical.

\subsection{Sensitivity to parameter values}

After the validation of the mechanical module of TWIG (Appendix B), with considering woods as elastic materials, the sensitivity to values of the used parameters have been checked, specifically the angle of sector of tension wood, Young's modulus values, $\Delta \beta$ values, and eccentricity. 
The effect of the value of each parameter has been tested for a range of values that can be observed in experiments (Table1), all other parameters kept constant (central value). The effect of the value of each parameter on the induced variation of curvature has been computed. The results are shown on figure 8 . They concern a cross section located at the stem base. The influence of these parameters has then been ranked to check the most influencing parameters. Then the influence of viscoelasticity has been checked.

\subsubsection{Effect of Young's modulus}

Increasing the values of the ratio between the Young's moduli of tension wood and normal wood increases the curvature linearly (Figure 8A). Increasing the values of wood Young's moduli whilst, keeping the difference constant, increases the curvature in a logarithmic manner (Figure $8 \mathrm{~B}$ ). This is due to the fact that the internal tensile force which causes the bending moment is governed by the Young's modulus of the tension wood multiplied by the maturation strain: the higher the Young's modulus, the higher the bending moment hence the curvature.

\subsubsection{Effect of the maturation strains of wood}

Increasing the values of maturation strains between the normal wood and the tension wood, increases the values of curvature linearly (Figure $8 \mathrm{C}$ ). Increasing the ratio of maturation strains while keeping the same difference between them in normal and tension wood, increases the values of curvature (figure 8D). The results are thus very sensitive to the values of maturation strain and particularly sensitive to the difference of maturation strains of normal and tension wood. Similarly to the previous section, this trend is due to the fact that the internal tensile force which causes the bending moment is governed by the maturation strain in the tension wood multiplied by the Young's modulus: the higher the maturation strain, the higher the bending moment hence the curvature.

\subsubsection{Effect of eccentricity of radial growth}


In order to investigate the influence of the eccentricity of the radial growth on the curvature, simulations were carried out for different values of the eccentricity $\delta$ written as follows: $\delta=k\left(R-R_{i n i}\right)$, where $R \in\left[R_{i n i}, R_{f i n}\right]$ is a given value of the stem radius and $k$ is a parameter to be varied between 0 and 1 to scan all possible values for $\delta$ (see Figure 5). Indeed, for fixed values of $R$ and $R_{i n i}$, an increase in the eccentricity leads to an increase of the proportion of reaction wood in the growing section, leading therefore to an increase in the bending moment caused by the differential maturation strains between reaction wood and normal wood. Simulation results are presented in Figure $8 \mathrm{E}$; it is seen that the curvature varies linearly in terms of the $k$ parameter, i.e. of the eccentricity $\delta$. This linear dependence was not expected. Indeed the variation of the first moment of area is the product of a distance (linear function of $\delta$ ) and of an area (also a function of $\delta$ ),and the curvature depends on the second moment of inertia (see Appendix A4) which is a non linear function of eccentricity (function of $\delta^{2}$ ). In the present case, the linear relationship between the curvature and the $k$ parameter is due to a compensation effect between the evolution of geometrical characteristics and the choice of wood mechanical properties (moduli of elasticity, maturation strains) used in the simulation. Generally speaking, this relationship might not be linear.

\subsubsection{Effect of the angle of the sector of tension wood.}

The curvature increases with increase of the tension wood sector in a non-linear fashion (Figure 8F). In this case, an increase of the tension wood leads to a non-linear variation of the first moment and therefore to a non-linear variation of the curvature as shown in Figure 8F.

\subsubsection{Effect of the kinetic of maturation in the layers}

Experiments have shown that a delay exists between the kinetics of radial growth and curvature in a tilted trunk, which could be due to the process of maturation in progress in the 
successive layers [12]. In this section, the model is used to investigate this hypothesis. Two cases are considered for this purpose.

Case 1: It is assumed that the axial shrinkage occurs in a new layer at the time of deposition of the layer, i.e. instantaneously, corresponding to the beginning of the current step. This is equivalent to consider that only the last added layer is maturing during the calculation step. Under this assumption, the final curvature at the stem base is about $0.026 \mathrm{~m}^{-1}$.

Case 2: Alternatively, it is considered that the maturation is in progress in all layers simultaneously i.e. the shrinkage is progressing in the successive layers since time of deposition till the end of the growing season. Due to the lack of knowledge about the process of maturation in the successive layers, the following hypotheses are used: 1) the maturation follows a parabolic law in the course of time, 2) the value of the maturation strain in the outermost layer is set equal to the value measured at the end of the growing season (central values of rlms in Table 1). Under these assumptions, the final curvature at the stem base which is obtained is about $0.050 \mathrm{~m}^{-1}$. Considering the maturation as a non instantaneous process doubles the value of final curvature.

\subsubsection{Ranking of parameters}

In order to rank the effect of each parameter on the predicted variation of curvature, the values of parameters have been normalised to give a range from 0 to $100 \%$. The variation of curvature was set to start from 0 . The results of the normalisation are shown on Figure 9. It suggests that the most influencing parameter is the difference of maturation strains. This is in agreement with what was found by Alméras and collaborators [18]. Next in importance is the angle of tension wood sector (i.e the amount of tension wood at any given cross-section) and finally the values of Young's modulus and eccentricity. The difference of Young's moduli has more influence on the variation of curvature than the values of Young's moduli per se.

\subsubsection{Influence of the material viscoelastic parameters}


In order to estimate the influence of the rheological effects on the process of reorientation of the trunk, the analysis is repeated for various values of the material parameters. The results are presented in Figure 10. Figure 10A shows that the final curvature increases as a quadratic function of the ratio between the Young's modulus $E_{1}$ of the viscoelastic branch in the Zener model and the Young's modulus $E_{0}$ of the elastic branch. When the relaxation time $\left(\alpha_{1}^{-1}=\frac{\eta_{1}}{E_{1}}\right.$, see Eq. (3)) in the Zener model is increased up to 50 days (Figure 10B), the delayed curvature (i.e. the part of the curvature developing in the course of time due to rheological effects in the growing stem) becomes negligible and the final curvature decreases rapidly to converge towards the values corresponding to perfect elastic materials.

\section{Comparison of simulations to experimental data}

In this last part, we present the shapes and variations of curvature obtained by simulations in comparison with shapes and variations of curvature measured experimentally during the gravitropic response of young poplar (Populus, I4551) artificially titled $35^{\circ}$ from the vertical. Simulations were first done with considering the wood as an elastic material and then considering the wood as a viscoelastic material.

\subsection{Simulations with wood considered as an elastic material - reference case}

Elastic behaviour has been chosen as reference case because of existing models to compare with and to assess validity of hypotheses. The predicted variations of curvature by the "elastic" model are compared to experimental data (Figure 11). To proceed to the validation of the mechanical module of TWIG, the regulation hypothesis has been replaced by introducing the data for the observed sectors of tension wood (angle, position).

The experimental data were obtained from a study of the re-erecting process of artificiallytilted young poplars. In the experiment the evolution of shape of the trunks was measured weekly during a vegetative season using 3D digitising technique [33]. The digitised shapes 
were then used to compute the temporal evolution of curvature fields along the gravitropic response, see [12] for the description of the computing method. The radial growth was also measured weekly at different locations along the trunks (approximately every $25 \mathrm{~cm}$, base of the shoot of the year). The trees were cut down at the end of the vegetative season to proceed with tension wood cartography. The sectors of tension wood were characterised by their sector angle. The radial growth increment under bark (because the influence of bark is considered negligible in computations) was obtained from measurements of radial growth on bark by removing the width of the bark considering that it did not change within the time of experiment. Measurements of residual maturation strain, wood density and longitudinal Young's moduli of normal- and tension- wood were taken from a similar experiment on the same poplar clone [32] (central values of Table I).

The curvature computed by the model and the curvature computed from experimental data have been compared considering two different hypotheses for the maturation process. A representative example of simulated and measured variations of curvature along the stem with considering the maturation process as instantaneous is shown on Figure 11A. The fields of curvature at three dates after inclination are traced: $t=0$ is the time of tilting, $t=2$ and $t=4$ correspond to two and four weeks after tilting. At $\mathrm{t}=0$ the experimental and simulated values of curvature are close. At $\mathrm{t}=2$ weeks and $\mathrm{t}=4$ weeks, there is a big discrepancy between experimental and simulated values of curvature. Simulated values are far smaller then experimental ones.

When the maturation process is considered non-instantaneous, the curvature between $\mathrm{t}$ and $\mathrm{t}+\mathrm{dt}$ is attributed to the effect of an increment of radial growth but not the one observed between $\mathrm{t}$ and $\mathrm{dt}$. To our knowledge there is no existing work that describes the kinematics of the maturation process and, hence the width of the active zone is unknown. Our hypothesis is that the duration of the maturation process is the same as the duration of the vegetative season 
so that all layers of cells are acting during all the righting-up process and that the maturation process follows a quadratic function. An example of comparison of simulated and computed variation of curvature along the trunk is shown on Figure 11B. The fields of curvature at three dates after inclination are traced: $\mathrm{t}=0$ refers to as the time of tilting, 2 weeks after tilting and 4 weeks after tilting. When considering that all the cell layers are maturing, the discrepancy between measured and simulated values is reduced: when considering the maturation process non instantaneous, the simulated values of curvature are twice fold higher than the simulated ones when considering the maturation process as instantaneous. Two weeks and four weeks after tilting the measured and simulated fields of curvature exhibit the same trend but the experimental values remains slightly higher than the simulated ones. Four weeks after tilting, there is an important discrepancy between the experimental and simulated values of curvature. One reason for this discrepancy is that the radial growth between the $2^{\text {nd }}$ and $4^{\text {th }}$ weeks after tilting was about $0.33 \mathrm{~mm}$ so far smaller than during the 2 weeks after titlting (increment of radius was $1.38 \mathrm{~mm})$.

Two other reasons can explain the discrepancy between simulated and computed data: i) in the model the maturation strains of wood are assumed to be constant, whereas there is experimental evidence that trees regulate their maturation prestresses [12]; ii) green wood is known to be viscoelastic and not elastic and this will have an effect on the reorientation of trees. We thus end this work by checking the effect of viscoelasticity on the simulated gravitropic response of tree.

\subsection{Simulations considering the wood as a viscoelastic material}

\subsubsection{Parameter values}

The parameter values are the same than those used for simulations in the frame of elasticity (central values, see Table1) except for the data concerning the rheological properties of wood: 
Young's modulus values are taken from available experimental measured values [32]: for normal wood, bending tests give a value of $2 \mathrm{GPa}$ assumed as $1 \mathrm{GPa}$ in each branch of the Zener's model; for tension wood, bending tests give a value of $4 \mathrm{GPa}$ assumed as $2 \mathrm{GPa}$ in each branch of the Zener model.

The analysis spans a 105 days period of growth. This period is divided in 15 elementary growth steps i.e. one step of growth per week. Each time step is subdivided in 20 short time intervals suitable for the viscoelastic analysis. As explained before, preliminary calculations have shown that this feature is required to ensure the accuracy of the analysis.

\subsubsection{Analysis of the reorientation process of the main axis}

Figure $12 \mathrm{~A}$ presents the result of a simulation of the evolution of the overall geometry of the poplar at successive calculation times. The simulated events are as follows:

- the poplar is inclined at an angle of $35^{\circ}$ from the vertical. First, the stem bends down towards the ground because of its self weight; during the next days, the bending down increases due to creeping of the stem;

- a thin layer of wood forms during the next growth step, with tension wood on the upper face of the stem. Due to higher shrinkage value in the tension wood, the stem bends up and the process of reorientation begins. The bending up increases with time because of creep.

On the figure, the red lines correspond to growth steps and the green lines represent the tree shapes at different viscoelastic steps. The data reveals that the introduction of viscoelasticity within the model leads to an increase of the righting-up process between two steps of growth. It is also noticeable that the variation of curvature between steps of growth increases with time. 
Figure 12B compares the successive computed shapes of a tree considering the wood as an elastic material (grey lines) or a viscoelastic material (dark lines). The simulation has been made with 5 steps of growth (covering a period of 105 days) instead of 15 steps of growth as in Figure 12A. Just after tilting, the computation which includes viscoelasticity leads to a sagging of the trunk. After the first step of growth:

- if we consider wood as elastic, the tree sags because tension wood production is not sufficient to counterbalance the self-weight increase due to radial growth;

- if we consider wood as viscoelastic, the tree rights-up.

After the second step of growth, the tree rights-up in the both cases but the difference between the computed curvatures increases with time. It is concluded that taking wood viscoelasticity into account leads to an acceleration of the righting-up process and consequently to an acceleration of the gravitropic response of the tree.

\subsubsection{Evolution of the stress distribution within a cross-section}

Figure 13 shows the evolution of normal stress distribution in a transverse cross section, at the initial state just after tilting, then 5 weeks, 10 weeks, and 15 weeks later (final state). In the initial state, the linear stress distribution is in accordance with the case of a section subjected to a normal force and a bending moment resulting from the self-weight of the trunk only. Then, the effect of the normal force and the bending moment due to the actual weight, combined with the effect of the differential shrinkage between normal wood and tension wood is observed. The shrinkage in the tension wood causes compression in the rest of the crosssection and creates an additional bending moment. This moment has a significant influence on the geometry by rotating the sections. Asymmetry in the stress distribution is due to the differential shrinkage between tension wood (located at the top of the figure) and normal wood (located at the bottom of the figure), as well as to the difference in elastic moduli. The 
stress gradient through the cross-section is steadily increasing in the course of time, due to the development of self-equilibrating stresses resulting from the setting of additive wood layers and production of tension wood on the upper side of the trunk. Progressive compression of the trunk heart is counterbalanced by progressive tension at its periphery with higher stress values on the upper side where the tension wood is developing.

\section{Conclusions}

The model developed in this paper is designed to simulate the successive shapes of a tree axis during the gravitropic response at the intra-annual scale (week). It takes into account the composite and heterogeneous nature of wood in a reorientating axis, introducing explicitly the differences of mechanical properties between normal and tension wood. The model takes also into account possible eccentricity of radial growth. The model has been validated from a mechanical point of view. At first, woods within the growing tree were considered as elastic materials. A sensitivity study of the model parameters has been done. It enabled to rank the influence of parameters on the predicted variations of curvature. The ranking of parameters showed that the most influential parameter is the value of maturation strains, followed by the angle of tension wood sector and finally by the modulus of elasticity of the woods. It also appears that an important influencing parameter could be the kinetic of maturation in the successive layers during the growing season. Adding the viscoelastic behaviour reveals that the righting-up process is significantly influenced in the course of time by the time-dependent properties of the materials; this could explain the fact that the righting-up process of trees can be observed even when radial growth is very limited. It appears that viscoelasticity of green wood plays an important role within the righting-up process and on the associated stress fields for retardation time up to 50 days. This leads to the conclusion that the values of viscoelastic parameters should be measured for a range of reaction and normal woods. Further work is 
therefore necessary to obtain experimental values of viscoelastic characteristics of green wood in order to quantify its importance on the gravitropic response of tree axes.

Comparisons with experimental data show that the observed values of curvature variations fall within model predictions based either on the assumption that only the last-produced layer of cells is maturing or all layers of cells are maturing. Further work appears now necessary on the wood maturation process. In particular, it is clear that the kinetics of the maturation zone as well as the regulation of maturation prestress levels are critical mechanisms to be elucidated.

The model presented in this paper enables to compute the successive mechanical states of a growing structure composed of viscoelastic materials and to predict the associated shape changes. In this paper, this approach has been applied to the case of the righting-up process of an axis in trees. To our knowledge this is the first time that the effect of wood viscoelasticity on the reorientation process of stems is examined. Obvious similarities between this phenomenon and the time-dependent behavior of concrete structures have been exploited in the setting of the mechanical model. Although the construction of bridges by incremental launching is nowadays a well established technology, the observation of the mechanisms of reorientation in trees could be profitable in leading to innovative methods for correcting critical stress or strain states in existing structures by introducing additional internal forces by means of smart materials, such as shape memory alloys for example.

\section{Acknowledgements}

This work was partly funded by a Massif Central project Convention FEDER: $\mathrm{N}^{\circ} 5306$

Convention FNADT: $\mathrm{N}^{\circ} 775-03$ and the ANR (Agence Nationale de la Recherche), Woodiversity project ANR-05-BDIV-012-03. 


\section{Bibliography}

[1] R.K. Bamber, The origin of growth stresses: A rebuttal, IAWA Bull. 8 (1987) 80-84.

[2] J.D. Boyd, Tree growth stresses. Evidence of an origin in differentiation and lignification, Wood Sci. Tech. 6 (1972) 251-317.

[3] L. Goswami, J.W.C. Dunlop, K. Jungnikl, M. Eder, N. Gierlinger, C. Coutand, G.Jeronimidis, P. Fratzl, I. Burgert, Stress generation in the tension wood of poplar is based on the lateral swelling power of the G-layer, The Plant J. 56 (2008) 531-538.

[4] G. Scurfield, A.B. Wardrop, The nature of reaction wood. VI. The reaction anatomy of seedlings of woody perennials, Aus. J. Bot. 10 (1962) 93-105.

[5] G. Casperson, Über die reaktionholzbildung und krümmungsbewegungen in asten, Ber. Deutsch Bot. Ges. 79 (1966) 121-133.

[6] J. Firn, R.D. Digby, The gravitropic set-point angle (GSA): the identification of an important developmentally controlled variable governing plant architecture, Plant Cell Environ. 18 (1985) 1434-1440.

[7] B. Moulia, C. Coutand, C. Lenne, Posture control and skeletal mechanical acclimation in terrestrial plants: implications for mechanical modelling of plant architecture, Am. J. Bot, 93 (2006) 1477-1489.

[8] E. W. Sinnott, Reaction wood and the regulation of tree form, Am. J. Bot. 39 (1952) 69-78

[9] R.R. Archer, B.F. Wilson, Mechanics of the compression wood response. I Preliminary analyses, Plant Physiol. 46 (1970) 550-556.

[10] R.R. Archer, B.F. Wilson, Mechanics of the compression wood response. II on the location, action, and redistribution of compression wood formation, Plant Physiol. 51 (1973) $777-782$.

[11] M. Fournier, H. Baillères, B. Chanson, Tree biomechanics: growth, cumulative prestresses, and reorientations, Biomimetics, 2 (1994) 229-251. 
[12] C. Coutand, M. Fournier, B. Moulia, The gravitropic response of poplar trunks: key roles of prestressed wood regulation and the relative kinetics of cambial growth versus wood maturation, Plant Physiol. 144 (2007) 1166-1180.

[13] T. Almeras, M.Derycke, G. Jaouen, J.Beauchêne, M. Fournier, Functional diversity in gravitropic reaction among tropical seedlings in relation to ecological and developmental traits, J. Exp. Bot. 60 (2010) 4397-4410.

[14] B. Jourez, T. Avella-Shaw, Effet de la durée d'application d'un stimulus gravitationnel sur la formation de bois de tension et de bois oppose dans de jeunes pousses de peuplier (Populus Americana cv 'Ghoy’), Ann. For. Sci. 60 (2003) 31-41.

[15] H. Yamamoto, M. Yosida, T. Okuyama, Growth stress controls negative gravitropism in woody plant stems, Planta 216 (2002) 280-292.

[16] T. Fourcaud, F. Blaise, Numerical modelling of shape regulation and growth stresses in trees. II. Implementation in the AMAPpara software and simulation of growth, Trees 31 (2003)131-148.

[17] T. Fourcaud, P. Lac, Numerical modelling of shape regulation and growth stresses in trees. I. An incremental static finite element formulation, Trees 17 (2003) 23-30.

[18] T. Almeras, B. Thibaut, J. Gril, Effect of circumferential heterogeneity of wood maturation strain, modulus of elasticity and radial growth on the regulation of stem orientation in trees, Trees 19 (2005) 457-467.

[19] T. Almeras, M. Fournier, Biomechanical design and long-term stability of trees: Morphological and wood traits involved in the balance between weight increase and the gravitropic reaction,J. theor. Biol. 256 (2009) 370-381.

[20] M. Fournier, A. Stokes, C. Coutand, T. Fourcaud, B. Moulia, Tree biomechanics and growth strategies in the context of forest functional ecology, in: A. Herrel, T. Speck, N. P. 
Rowe (Eds.), Ecology and biomechanics. A mechanical approach to the ecology of animals and plants, Taylor and Francis, Boca Raton, London, New-York, 2006, pp. 1-33.

[21] J. Gril, B. Thibaut, E. Berrada, G. Martin, Recouvrance hygrothermique du bois vert. I. Influence de la temperature. Cas du jujubier (Ziziphus lotus (L) Lam.), Ann. For. Sci. 50 (1993a) 57-70.

[22] J. Gril, B. Thibaut, E. Berrada, G. Martin, Recouvrance hygrothermique du bois vert. II. Variations dans le plan transverse chez le châtaignier et l'épicéa et modélisation de la fissuration à coeur provoquée par l'étuvage, Ann. For. Sci. 50 (1993b) 487-508.

[23] C.J. Hogan, K. J. Niklas, Temperature and water content effects on the viscoelastic behaviour of Tilia Americana (Tiliaceae) sapwood, Trees 18 (2004) 339-345.

[24] J-F. Destrebecq, B. Jurkiewiez, A numerical method for the analysis of rheologic effects in concrete bridges, Comput. Aided Civ. Infrastruct. Eng. 16 (2001) 347-364.

[25] P. Gwożdziewicz, B. Jurkiewiez, J-F. Destrebecq, Modelling of time effects in prestressed concrete bridges, in: FIB (Eds), Fib Symposium. Structural Concrete. The Bridge between People, Viacon Agency, Prague, 1999, pp. 455-461.

[26] B. Jurkiewiez, J-F. Destrebecq, A. Vergne, Incremental analysis of time-dependent effects in composite structures. Computers and Structures, 73 (1999) 425-435.

[27] J. Lemaitre, J-L. Chaboche, Mechanics of solid materials, Dunod, Paris, 1988.

[28] J.L. Grossiord, G. Couarraze, Initiation à la rhéologie, 2nd ed., Lavoisier, Paris, 1991. [29] W. Nowacki, Théorie du fluage, Eyrolles, Paris, 1965.

[30] J. Mandel, Théorie générale de la viscoélasticité linéaire, Cahier du Groupe Français de Rhéologie, 3 (1958) 21-35.

[31] Z.P. Bazant, S.T. Wu, Rate type creep law of aging concrete based on Maxwell chain, Materials and Structures, 37 (1974) 45-60. 
[32] C. Coutand, G. Jeronimidis, B. Chanson, C. Loup, Comparison of mechanical properties of tension and opposite wood in populus, Wood Sci. Technol. 38 (2004) 11-24.

[33] H. Sinoquet, B. Moulia, R. Bonhomme, Estimating the three dimensional geometry of a maize crop as an input of radiation models: comparison between three dimensional digitizing and plant profiles, Agric. For. Meteorol. 55 (1991) 233-249.

[34] R.R. Archer, Growth stresses and strain in trees, Springer-Verlag, Berlin, 1986.

[35] S. Laroze, Mécanique des structures. Tome2 : Poutres, $2^{\text {nd }}$ ed., Masson, Paris, 1988.

[36] K.J. Niklas, The mechanical role of Bark, Am. J. Bot., 86 (199) 465-469. 


\section{Appendices}

\section{Appendix A}

\section{The mechanical module of TWIG}

From a mechanical point of view, growth can be considered as successive additions of new material on a structure with time. In this model axial growth of the year shoot is not taken into account (being of negligible mass), but could be added if necessary by incrementally increasing the mass at the tip of the trunk; growth of lateral branches could be included via additional distributed masses along the trunk; radial growth is simulated by successive additions of new rings of material on the initial geometry of the trunk.

\section{A.1 Growing axes in trees: a non classical engineering problem}

Axes of trees are very often slender structures, typically assimilated to cantilever beams, with diameter/length ratio $<1 / 20$. However, as explained by Fournier et al. [11], the computation of the mechanical state of a growing tree axis differs from classical engineering problems in that the geometry of the axis and its mass change with time [34]. Because of growth, new material is added on an initial pre-existing structure so "that the structure is loaded as by continuously changing forces while new tissue differentiates progressively" [11]. Because of the variable geometry and mass in the problem [11], this requires an approach with incremental steps. It follows that:

- the problem is defined and solved at the cross section scale using classical beam theory between two times ( $t$ and $t+d t)$, so long as growth remains infinitesimal during that time increment;

- the mechanical state of the cross section at a given time must be computed by superimposing all the previous increments of mechanical state. 
To summarise, a growing axis exhibiting gravitropic response is a beam loaded by two forces, the increment of self-weight (growth), which tends to bend the axis downwards, and the internal forces due to the asymmetrical maturation of wood (gravitropic response), which tends to bend the axis upwards. Figures $2 \mathrm{~A}$ and $2 \mathrm{~B}$ show the decomposition of these loads into statically equivalent forces and moments. The self-weight induces (Figure 2A) a bending moment around $\mathrm{X}$, a normal force along $\mathrm{Z}$ and a shear force along $\mathrm{Y}$. The asymmetrical wood maturation process induces (Figure 2B) a bending moment around $\mathrm{X}$ and a normal force along Z.

In the case of slender beam structures the Navier-Bernouilli's principle allows to neglect transverse shear effects [35]. In this model only longitudinal stresses and strains are considered, neglecting also radial and circumferential components.

Between two successive time steps, the relationship between axial strain, curvature, axial force and bending moment is obtained from force and moment equilibrium at a given cross section, resulting in the following equation:

$\left(\begin{array}{c}\Delta \varepsilon \\ \Delta \chi\end{array}\right)=[S]\left(\begin{array}{c}\Delta N_{z} \\ \Delta M_{x}\end{array}\right)=\left[\begin{array}{ll}S_{11} & S_{12} \\ S_{12} & S_{22}\end{array}\right]\left(\begin{array}{c}\Delta N_{z} \\ \Delta M_{x}\end{array}\right)$

where $\Delta \varepsilon$ and $\Delta \chi$ are the variations of axial strain and curvature between $t$ and $t+d t,[S]$ is the compliance matrix used in the time step and $\Delta N$ and $\Delta M$ the variations in axial force and bending moment as a function of time $(\mathrm{t})$ and position along the stem $(\mathrm{Z})$. The compliance coefficients in the $[\mathrm{S}]$ matrix are given by inverting the stiffness matrix.

\section{A.2 Wood tissue heterogeneity}

In our work, the axis is composed of two materials, normal- and tension-wood considered homogeneous at the "tissue" scale: we do not take into account any ultra-structural features of tissues (e.g. fibres, vessels) or of the cell components (e.g. cell wall, plasma-membrane, cytoplasm). 
The only tissue considered here is the wood: the bark (real bark and phloem tissue) is ignored in the model because, even if located at the periphery, its stiffness is negligible compared to that of wood until the bark thickness is $32 \%$ that of wood and its stiffness is $50 \%$ that of wood [36]. This is not the case of young poplars where the bark thickness was about $10 \%$ that of wood.

\section{A.3 Eccentricity of radial growth}

In this paper we treated the case of cross sections exhibiting eccentricity of radial growth but remaining circular (Figure 5). The eccentricity of radial growth is taken into account by the variable $(\delta)$ which is the distance between the pith and the geometrical centre of the cross section (Figure 5). It is an input of the model.

The increment of radius, $\Delta r(\theta)$, for each time step, has been computed as a function of the eccentricity $\delta$ and the position of the current point on the cross section defined by the angle $\theta$ in Figure 5. The relationship between $r(\theta)$ and $\delta$ is given by:

$$
r(\theta)=\delta \sin \theta+\sqrt{R^{2}-\delta^{2} \cos ^{2} \theta}
$$

\section{A.4 Consequences of eccentric radial growth and wood heterogeneity on the mechanical equilibrium of the cross section}

In bending of beams which possess mirror symmetry of geometry and material properties with respect to the geometrical mid-plane of the cross-section, the neutral axis coincides with the centroidal axis of the beam and, as a consequence, the compliance matrix is diagonal (Equation A3).

For this special case equation (A1) becomes: 


$$
\left(\begin{array}{c}
\Delta \varepsilon_{z} \\
\Delta \chi
\end{array}\right)=\left[\begin{array}{cc}
S_{11} & 0 \\
0 & S_{22}
\end{array}\right]\left(\begin{array}{l}
\Delta N_{z} \\
\Delta M_{x}
\end{array}\right)
$$

where, at the given cross section at a distance $z$ from the origin ( $\mathrm{z}=0$ at the base of the trunk), $\varepsilon_{\ell}$ is the axial normal strain, $\chi$ the curvature, $M_{X}$ the bending moment, $N_{Z}$ the normal force.

In our case, the eccentric radial growth and the asymmetrical sector of reaction wood within the cross section both influence the mechanical state of the cross section. Indeed, in this case there is a coupling between bending moment and normal axial strain. In other words, when a non-symmetrical structure is submitted to bending, it generates bending strains but also additional longitudinal strain. Similarly, applying a normal force to a non-symmetrical structure leads to bending strains as well as to direct normal strain. As a result, the neutral axis of bending does not coincide anymore with the mid-plane of the section, or centroidal axis, the bending moment induces a normal axial strain, the normal force induces a bending moment and the compliance matrix is not diagonal.

In this case Equation (A3) becomes:

$$
\left(\begin{array}{c}
\Delta \varepsilon \\
\Delta \chi
\end{array}\right)=[S]\left(\begin{array}{l}
\Delta N_{z} \\
\Delta M_{x}
\end{array}\right)=\left[\begin{array}{ll}
S_{11} & S_{12} \\
S_{12} & S_{22}
\end{array}\right]\left(\begin{array}{l}
\Delta N_{z} \\
\Delta M x
\end{array}\right)
$$

Solving the problem consists in calculating the force vector terms and the terms of the compliance matrix in each step increment.

\section{A.5 Calculation of the forces acting on the beam: $M_{X}$ and $N_{Z}$}

The growing axis is submitted to two increments of load at each time step: an increment of self-weight and the asymmetrical maturation stresses of newly formed wood. These two acting loads result in an increment of bending moment $\Delta M_{X}$ and in an increment of normal force $\Delta N_{Z}$. These values are calculated as follows: 
- $\Delta M_{X}$ is the resulting bending moment and is the sum of the bending moment due to selfweight, $\Delta M_{X}^{S}$, and the bending moment due to the maturation phenomenon, $\Delta M_{X}^{\text {Mat }}$, which are of opposite signs:

$$
\Delta M_{X}^{\text {total }}=\Delta M_{X}^{S}+\Delta M_{X}^{M a t}
$$

- $\Delta N_{Z}^{\text {Total }}$, the resulting normal force, is the sum of the normal force due to the increment of self-weight and the maturation process:

$$
\Delta N_{Z}^{\text {Total }}=\Delta N_{Z}^{S}+\Delta N_{Z}^{\text {Mat }}
$$

For convenience and further validation tests, the model computes separately the loads due to self-weight and the loads due to the wood maturation process. The bending moment due to self-weight is considered as an "external" force acting on the beam and the load due to the maturation process is considered as an "internal" force resulting from the maturation process of normal- and reaction-wood.

\section{A.6 Bending moment and axial force due to increment of self weight:}

The bending moment at a given cross section $\mathrm{Z}$ is the sum of the bending moment moments due to the distal cross sections. To take into account the taper of axes, the problem has been discretised by considering the trunk as series of truncated cones. The volume of each cone is approximated by the volume of the cylinder of same height and radius equal to the mean radius and is given by $\pi \bar{R}^{2}(Z) \Delta Z$.

At each time step, the increment of bending moment, $\Delta M_{X}^{S}(i)$, at cross section $i$ due to the increment of self-weight due to the segments above has been computed from:

$$
\Delta M_{X}^{S}(i)=g \sum_{j=i}^{n} \Delta m_{j}\left(\sum_{k=i}^{n} l_{k} \cos \gamma_{k}\right)
$$


Where $\Delta m_{j}$ is the increment of mass added on each segment of the axis (i.e. the mass of the newly formed wood between two successive time steps), $g=9.81 \mathrm{~m} \cdot \mathrm{s}^{-2}$ is the acceleration of gravity, $\gamma_{k}$ the angle of inclination of the segment to the vertical and $l_{k}$ the length of a segment (which is constant along the axis in this work). In Equation (A.7), the terms in quotes correspond to the lever arm.

Normal forces due to self-weight are negligible and have been omitted in this model.

\section{A. 7 Bending moment and axial force due to asymmetrical wood maturation process}

As explained before, the asymmetrical composite structure and the eccentricity both lead to bending-stretching coupling so that the force vector has two components: a bending moment and a normal force.

Bending moment:

The bending moment due to the asymmetrical maturation of wood is the sum of the bending moment due to the maturation of normal wood (NW) and the bending moment due to the maturation of tension wood (TW):

$M_{X}^{M a t}=M_{X}^{M a t N W}+M_{X}^{M a t T W}$

$M_{X}^{M a t N W}$ and $M_{X}^{M a t T W}$ are calculated from:

$\left\{\begin{array}{l}M_{X}^{M a t N W}=\int_{A_{N W}} \beta_{N W} E_{N W} Y d A_{N W} \\ M_{X}^{M a t T W}=\int_{A_{T W}} \beta_{T W} E_{T W} Y d A_{T W}\end{array}\right.$

where $\beta_{\mathrm{NW}}$ and $\beta_{\mathrm{TW}}$ are, respectively, the residual longitudinal maturation strains for normal wood and tension wood; in this first version of the model, they are assumed to be constant.

$\underline{\text { Normal force }}$ 
Similarly, the normal force due to the asymmetrical maturation of wood is given by:

$$
\Delta N_{Z}^{\text {Total }}=\Delta N_{Z}^{\text {MatNW }}+\Delta N_{Z}^{M a t T W}
$$

$\Delta N_{Z}^{M a t N W}$ and $\Delta N_{Z}^{M a t T W}$ are computed from:

$$
\left\{\begin{array}{l}
N_{z}^{\text {Mat } N W}=\int_{A_{N W}} \beta_{N W} E_{N W} d A_{N W} \\
N_{z}^{\text {MatTW }}=\int_{A_{T W}} \beta_{T W} E_{T W} d A_{T W}
\end{array}\right.
$$

\section{A.8 Computation of the variation of curvature between $t$ and $t+d t$ :}

Using the principle of superposition, the total curvature of a cross section at a time $t+d t$ is taken as the sum of the curvature at time $t$, the increment of curvature due to the increment of self-weight between the times $t$ and $t+d t$, and the increment of curvature due to the maturation process in the wood formed between times $\mathrm{t}$ and $\mathrm{t}+\mathrm{dt}$ :

$$
\chi(t+d t)=\chi(t)+\Delta \chi^{S}(d t)+\Delta \chi^{M a t}(d t)
$$

For each segment the product of the compliance matrix (= inverse of stiffness matrix) and of the increment of loads vectors gives the terms of strain vectors due to the self-weight

$$
\left(\begin{array}{c}
\Delta \varepsilon^{S} \\
\Delta \chi^{S}
\end{array}\right) \text {, and those due to the wood maturation process }\left(\begin{array}{l}
\Delta \varepsilon^{M a t} \\
\Delta \chi^{M a t}
\end{array}\right) \text {. }
$$

\section{A.9 Inputs/Outputs of TWIG:}

The inputs of the model are:

- $\quad$ kinetics of radial growth (change in diameter with time step);

- mechanical properties of wood (Young's modulus of juvenile, normal- and reaction wood, residual longitudinal maturation strains of woods, wood densities);

- $\quad$ initial geometry of the trunk (length, taper and orientation). 
At each time step the outputs of the model are:

- the variation of curvature for any cross section of the axis;

- $\quad$ successive shapes of the axis;

- location and quantity of tension wood within the axis;

- the distribution of prestresses at any cross section.

\section{A.10 TWIG computer programme}

Matlab (7.2) for Windows, (The MathWorks@) was used for the whole programming of TWIG. The upper limit of the integrals in Equations A.9, A.11 contains the variable of integration $(\theta)$. The numerical computation is carried out by subdividing the areas of tension and normal wood into triangular area elements and adding up their contributions (Figure 14) which speeds up considerably the evaluation of the integrals. The analytical equations of each line delimiting each triangle are known. This enables us to calculate the areas, and their properties (first moment and second moment). Figure 14 shows the calculation of these properties for the tension wood zone. The values for zones Z1, Z2 and Z3 are calculated and then multiply by two in order to obtain the results for the tension wood. The same procedure is used for normal wood.

\section{Appendix B}

\section{Validation of the model from a mechanical point of view}

In order to check the validation of the model, some simple mechanical cases were studied: first we considered a homogeneous cylinder (radius of $0.009 \mathrm{~m}$ ), $1.5 \mathrm{~m}$ long, mass density of $1000 \mathrm{~kg} \cdot \mathrm{m}^{-3}$. We computed its deflection under its self-weight when tilted at an angle of $45^{\circ}$. The analytical calculation gives a bending moment equal to -1.9858 N.m and curvature $-0.1927 \mathrm{~m}^{-1}$. The model gives values of the bending moment at the stem base of -2.0257 N.m 
and a curvature value at the stem base of $-0.1966 \mathrm{~m}^{-1}$, resulting in an error about $2.0 \%$ which is pretty small.

In a second time we considered the same structure with a loading induced by the addition of new material on the trunk. The analytical computation gives a bending moment equal to -15.8866 N.m and the model gives a bending moment equal to -17.6 N.m which results in an error of $10 \%$. This error seems to be important but it is simply due to that the angle of the trunk has been taken as constant (for simplification of calculations) after the addition of new material on the trunk, which is not exact.

Finally, we tested the presence of tension wood sectors of about $90^{\circ}$ and $175^{\circ}$ and obtained errors of less than $1 \%$ in both cases. The results of these tests demonstrate the validity of the model from a mechanical point of view.

As the model uses a discretisation of the trunk into segments, the accuracy of the result will also depends on the number of segments. The values of variation of curvature with increasing the number of segments of trunk converge rapidly toward an asymptote. Considering a trunk of $1.5 \mathrm{~m}$ long, 1000 segments as the control case, taking 500 segments instead of 1000 led to an error less than 1\%; taking 100 segments instead of 1000 led to an error about $3 \%$ which is small. Thus, all simulations in the present work have been done by discretizing the stem into 100 segments of equal length, i.e. segments about $1.5 \mathrm{~cm}$ long. 


\section{Legends of Figures}

Figure 1: Representative example of experimental results: evolution of the shape of the trunk of a one year-old poplar tree after being artificially tilted of $35^{\circ}$ from the vertical During the two first weeks after tilting, the trunk sags. After this sagging phase, the tree curves up. After the $10^{\text {th }}$ of July (42 days after tilting), a decurving process starts at the top of the trunk and progresses towards the base of the trunk. As a result, at the end of August an important part of the trunk is back to the vertical position and straight.

Figure 2: Acting forces on a beam cross section composed of normal and tension wood The trunk represented by a composite beam is subjected to two kinds of loadings: the loading due to the increments of self-weight arising from the deposition of successive layers of wood and the loading due to the difference of axial shrinkage arising from the asymmetric production of tension wood within the cross section. Both loadings induce two internal forces: a normal force and a bending moment around the $\mathrm{X}$ axis.

\section{Figure 3: Expression of viscoelasticity phenomenon}

A: representation of the stress-relaxation phenomenon: when a sample is subjected to a constant strain, one can observe a decrease in the stress with time.

B: representation of the creep phenomenon: when a sample is subjected to a constant stress, one can observe an increase in the strain with time

Figure 4: Representations of a viscoelastic models

A: Generalized Maxwell's chain 
A generalized Maxwell's model is composed of $r+1$ branches: one branch is composed of a spring, all the other branches are composed of a spring linked in series to a dashpot. This type of model enables to simulate both creep and relaxation phenomena.

B : Zener's model used to simulate the viscoelastic behaviour of wood

A Zener's model is composed of two branches: one branch is composed of a spring, the second branch is composed of a spring linked to a dashpot (viscous).

\section{Figure 5: Scheme representing a growing cross section within the model}

The cross sections are circular. The initial core of the cross section is composed of normal wood. The successive layers of wood are composed of normal and tension wood. The tension wood sector within a layer is characterised by an angle $\theta$. The eccentricity of radial growth is quantified by the distance $(\delta)$ between the pith and the geometrical centre of the cross section.

\section{Figure 6: General flowchart of the numerical solution}

At the beginning of simulations, the geometrical characteristics of the trunk are given as well as the mechanical properties of wood and a law describing the tree radial growth. The model first computed the shape of the trunk just after tilting i.e. the shape due to self-weight. Then a step of growth takes place. The model computes the effect of acting forces on the trunk geometry which is updated. Then, the successive viscoelastic steps are carried out computing the geometry and stresses at each step. The structure and mechanical stresses are then updated before going to the next step of growth.

\section{Figure 7: Effect of the number of viscoelastic steps on the delayed curvature}

The figure shows the delayed curvature of a cross section located at the stem base, obtained with increasing the number of viscoelastic steps between two steps of growth. It appears that 
the curvature becomes stable after 20 viscoelastic steps. As a consequence, a value of 20 viscoelastic steps is retained for all further simulations.

\section{Figure 8: Sensitivity to parameters of the computed variation of curvature.}

A, B: influence of the values of Young's moduli of wood. In A, the ratio between the Young's moduli of normal and tension wood is increased. In B, the values of Young's moduli of normal and tension wood are increased whilst keeping the same difference.

C, D: influence of the values of maturation strains of woods. In C, the difference between the maturation strain in normal and tension wood is increased. In D, the values of the ratio of maturation strains in normal and tension wood is increased but the difference of the two values is kept constant.

E: influence of eccentricity

F: effect of the angle of tension wood

Figure 9: Ranking of the influence of parameters on the predicted variation of curvature.

To hierarchy the influence of parameters on the predicted variation of curvature, the values of parameters were normalised and the lowest values of the variation of curvature were all set to 0 . The most influencing parameter is the difference of the values of the maturation strains.

\section{Figure 10: Effect of viscoelastic parameters on the computed final curvature}

A: effect of the ratio of Young's moduli between the two branches of Zener's models. When increasing the ratio of Young's moduli, the final curvature increases following a quadratic function.

B: Effect of the viscosity on the computed final curvature. 
The effect of viscoelasticity is significant until the relaxation time (i.e. $\alpha_{1}^{-1}=\eta_{1} / E_{1}$ in the Zener's model, see Eq. (3)) associated with the viscosity reaches a value of 50 days.

Figure 11: Comparison of simulated and measured curvature fields with two different hypotheses concerning the maturation process

In each sub-figure the data represent the curvature field along the trunk, at three dates: $t=0$ is the time of tilting, $\mathrm{t}=2$ refers to two weeks after tilting, $\mathrm{t}=4$ refers to four weeks after tilting. The bold lines represent curvature computed from experimental records of tree shape, the dotted lines represent the simulated curvatures.

A: results obtained when considering the maturation process being instantaneous

B: results obtained when considering the maturation process lasting the whole vegetative season.

Figure 12: Example of simulation of the successive shapes of a tree with taking viscoelasticity of wood into account.

A) The figure illustrates the successive shapes of a tree during its gravitropic response with 15 steps of growth (red lines) and viscoelastic steps (green lines). The trunk shape varies between two steps of growth solely because of viscoelasticity phenomena.

B) The figure shows the successive shapes of a tree during its gravitropic response with 5 steps of growth considering wood as an elastic material (grey lines) or a viscoelastic material (black lines). For the computations where wood is considered as a viscoelastic material, only the final tree shapes after 20 viscoelastic steps in each growth step are shown. The successive shapes acquired by the trunk are numbered in their order of appearance during computation (grey numbers for wood as elastic, black numbers for wood as a viscoelastic material). With time, the differences between elastic and viscoelastic predictions increase. 
Figure 13: Profiles of the longitudinal stress in the median section of the trunk at various times after tilting

The distribution of stresses in a cross section just after tilting follows a classical linear shape for a homogenous loaded beam. Because of the addition of successive new layers of material the distribution of stresses becomes non linear and asymmetrical: all around the stem periphery the prestresses are tensions. The level of prestress is higher on the side were tension wood is produced (top of the figure). With time, the compression of the initial core of the tree increases.

Figure 14: Schematic representation of wood sectors for a rapid computing of moments of inertia within cross section. 


\begin{tabular}{|l|l|}
\hline \multicolumn{1}{|c|}{ parameter } & \multicolumn{1}{c|}{ Range } \\
\hline Young's modulus of tension wood (MPa) & $\begin{array}{l}2000 \text { to } 10000 \mathrm{MPa} \text {, central value }=4000 \\
\mathrm{MPa}\end{array}$ \\
\hline Young's modulus of normal wood $(\mathrm{MPa})$ & $\begin{array}{l}2000 \text { to } 10000 \mathrm{MPa} \text {, central value }=2000 \\
\mathrm{Mpa}\end{array}$ \\
\hline Eccentricity (\%) & 0 to $100 \%$, central value $=0$ \\
\hline rlms of tension wood & $5 \mathrm{E}-4$ to $5 \mathrm{E}-3$, central value $=11 \mathrm{E}-4$ \\
\hline rlms of normal wood & $5 \mathrm{E}-4$ to $5 \mathrm{E}-3$, central value $=5 \mathrm{E}-4$ \\
\hline Angle of sector of tension wood & $10^{\circ}$ to $175^{\circ}$, central value $=90^{\circ}$ \\
\hline
\end{tabular}

Table 1: ranges of values of the parameters used for sensitivity tests 


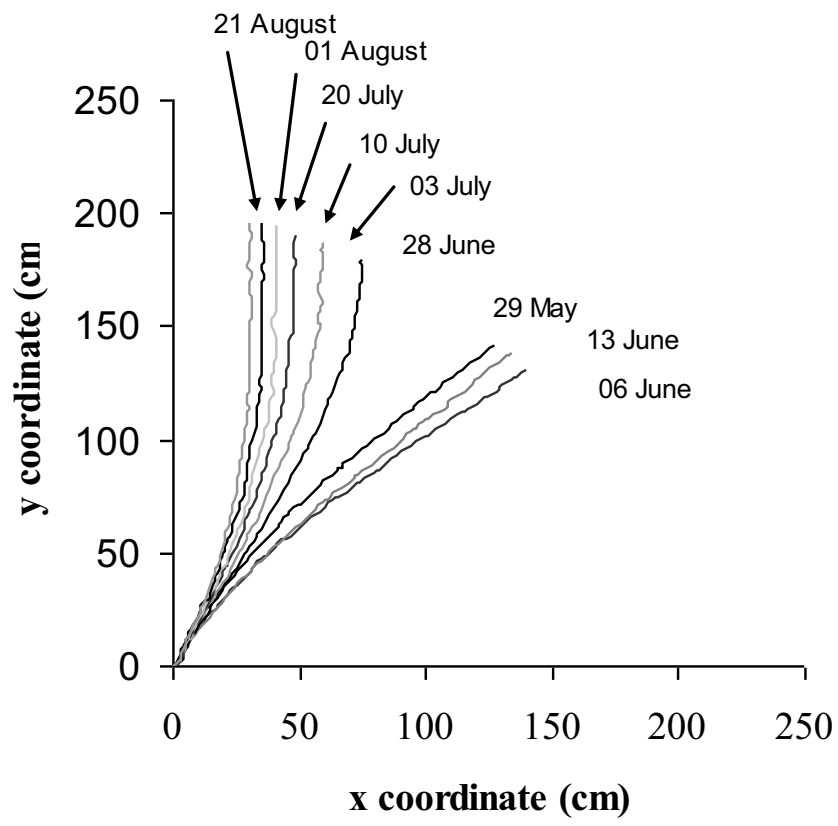

Figure 1 
Figure 2

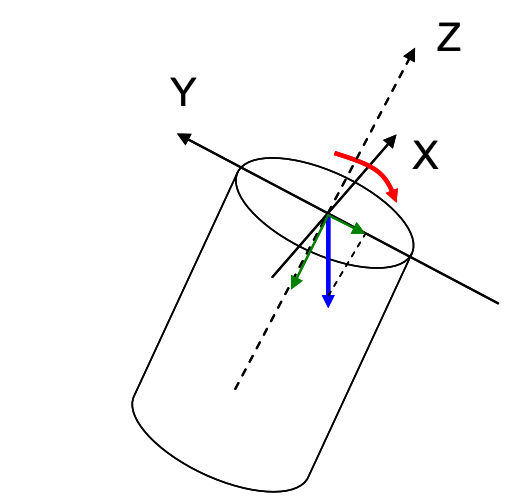

Increment of self-weight

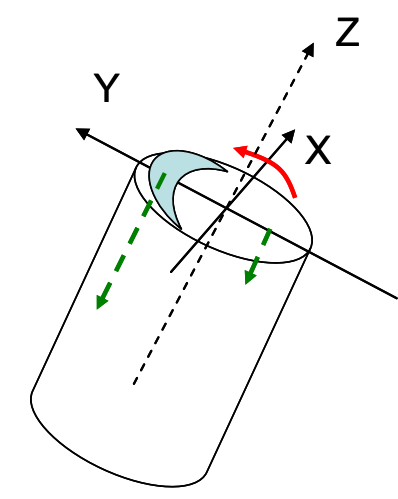

Effect of tension wood

Figure 2 
Figure 3
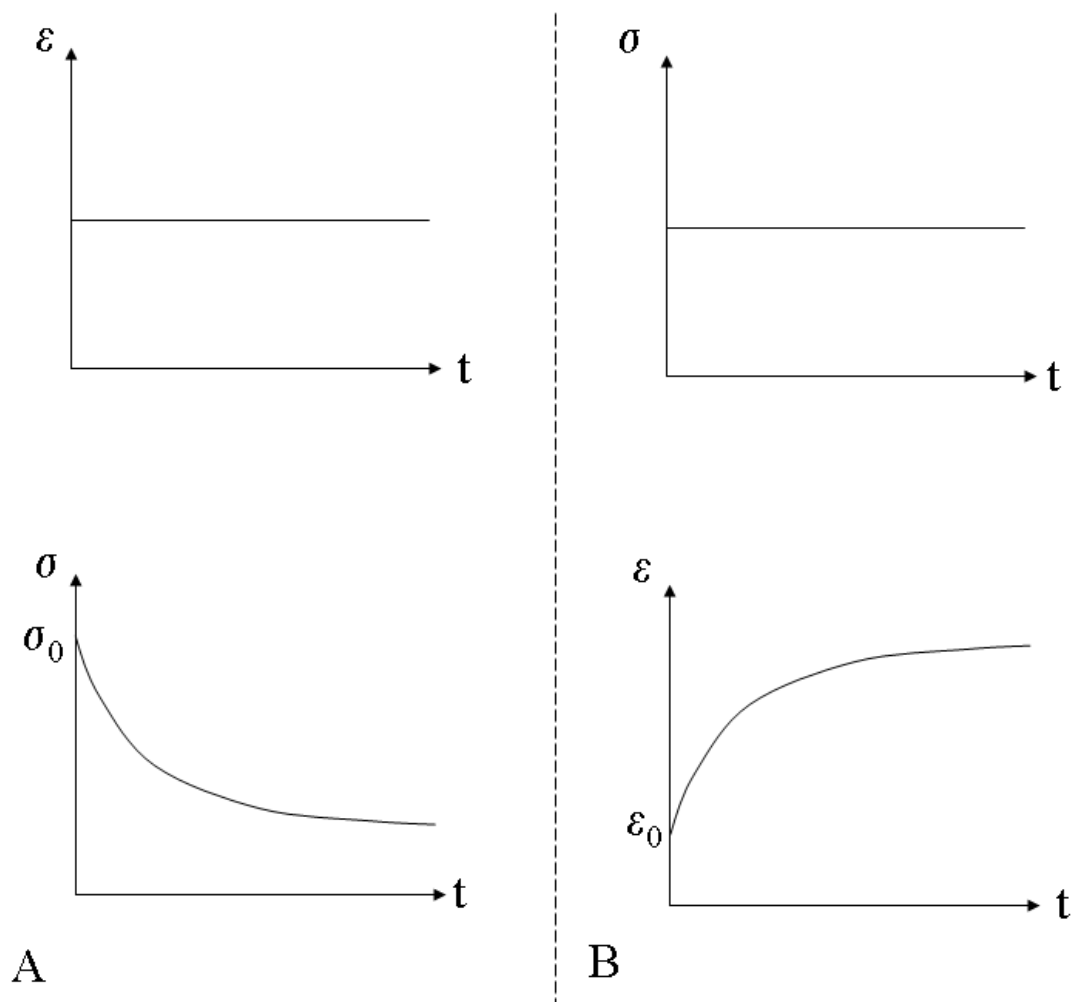

Figure 3 
A

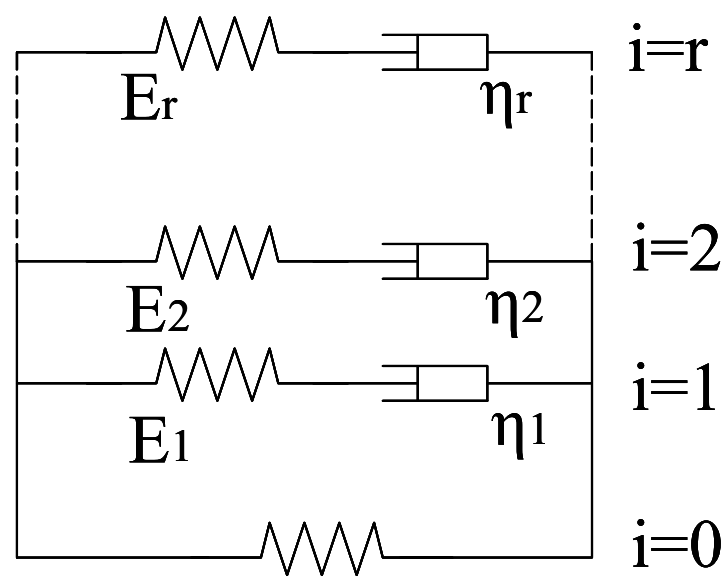

E0

B

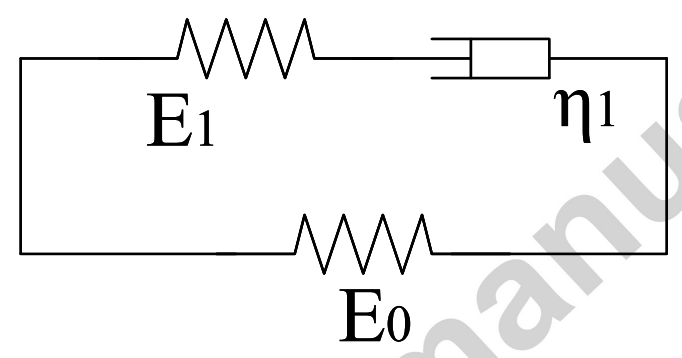

Figure 4 
Figure 5

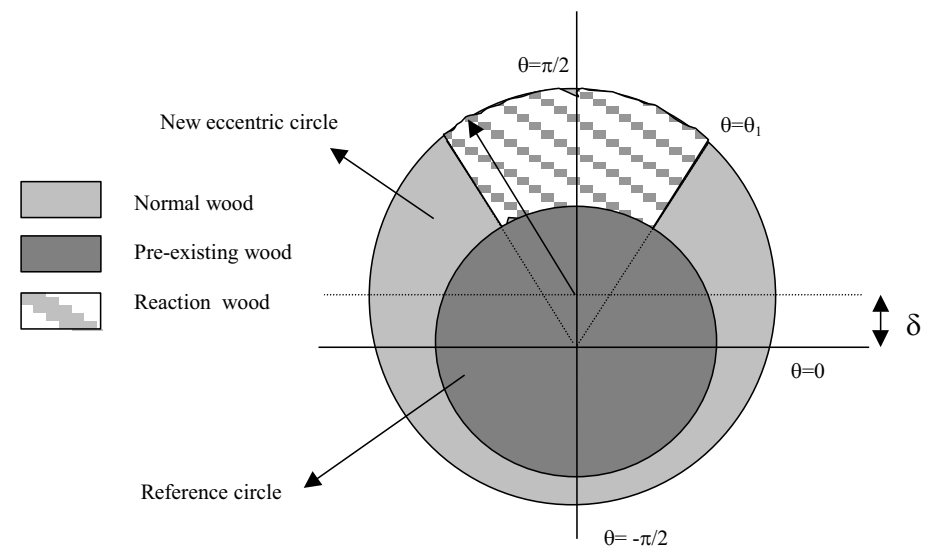

Figure 5 


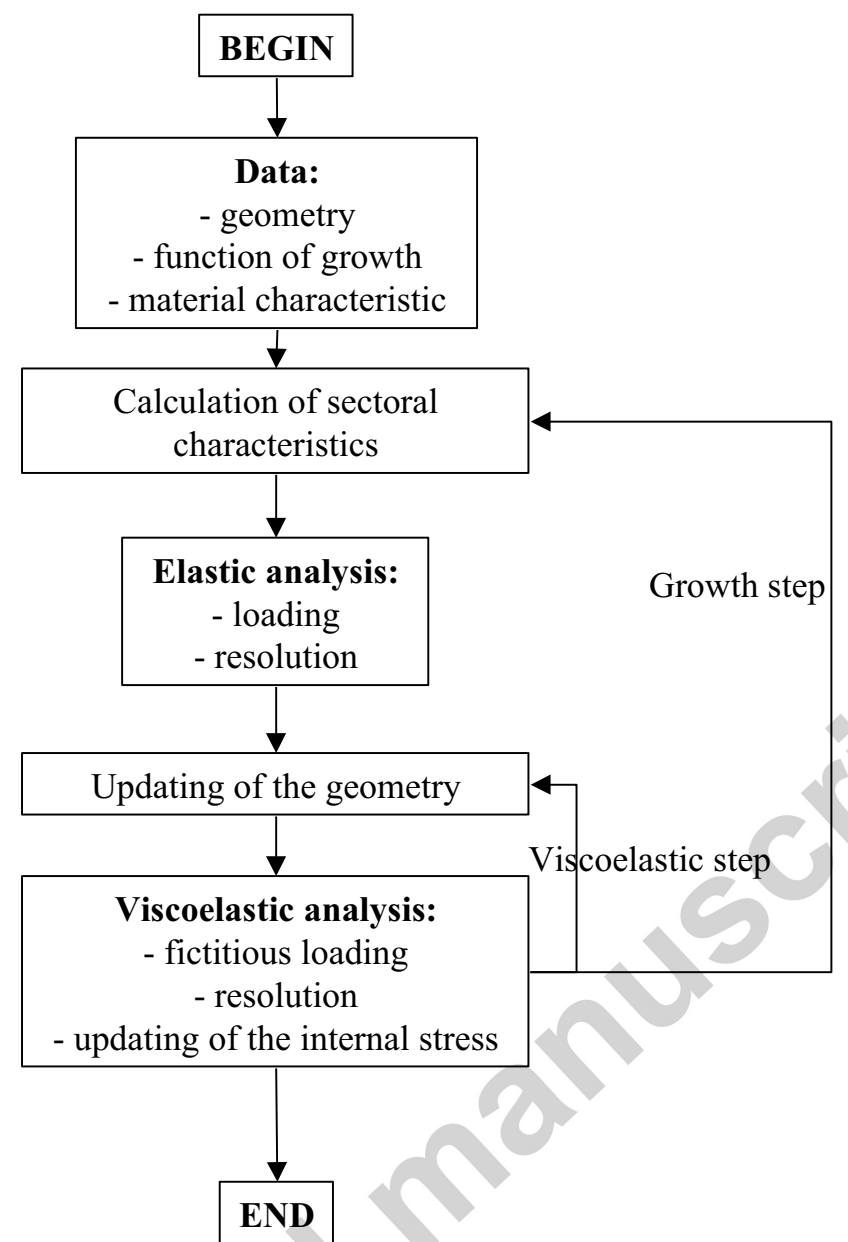

Figure 6 


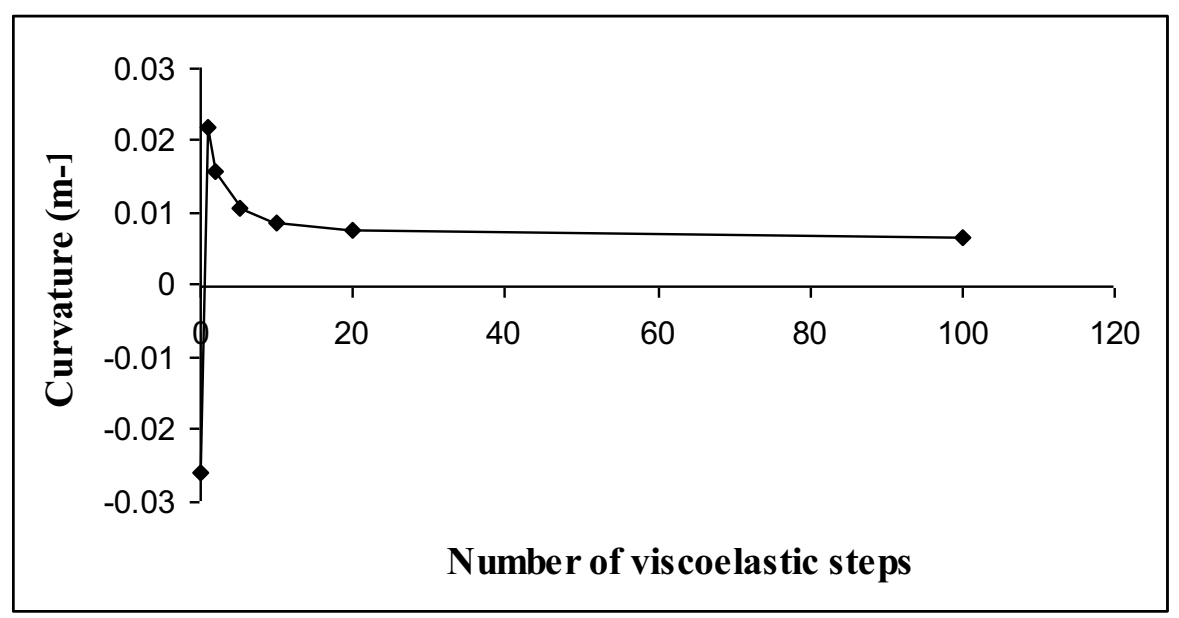

Figure 7 
1
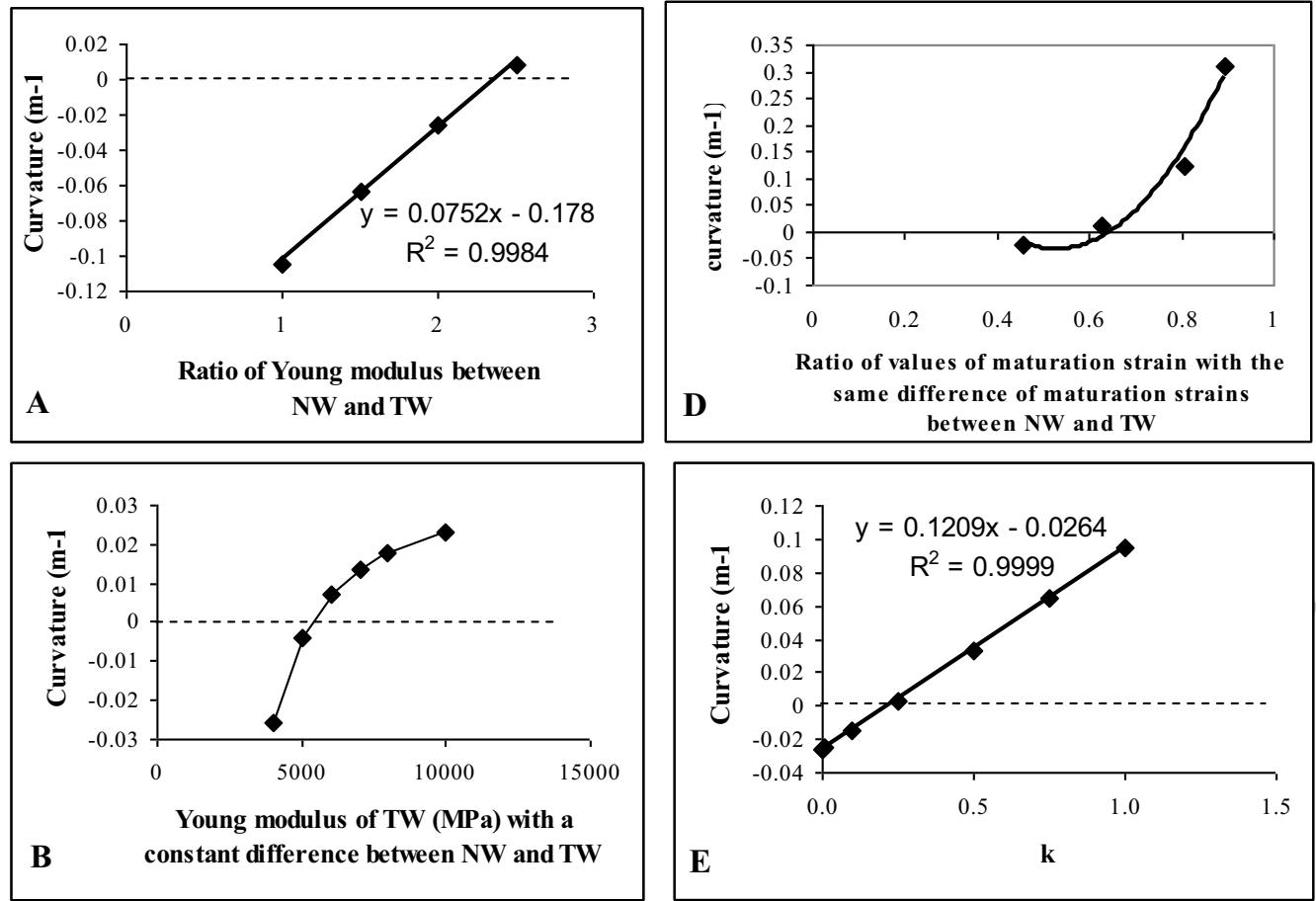

3
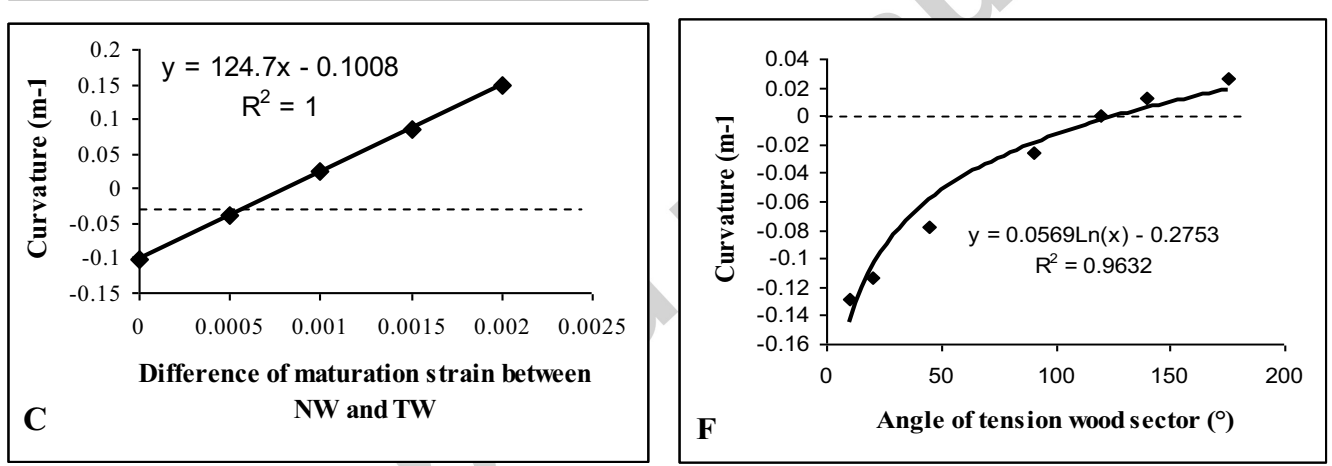

Figure 8 


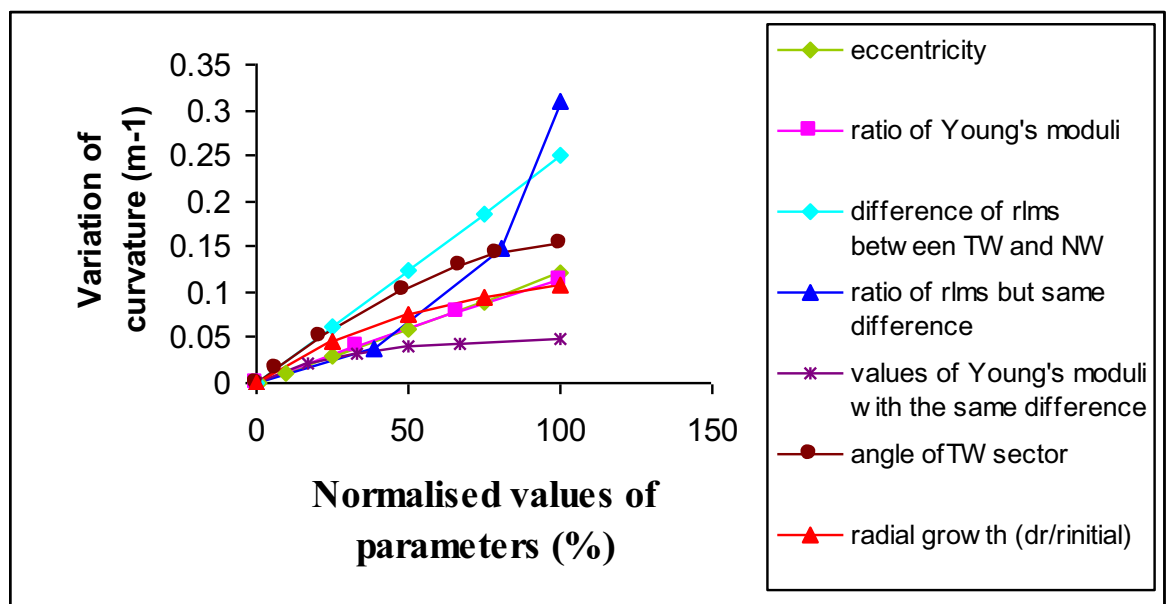

Figure 9 

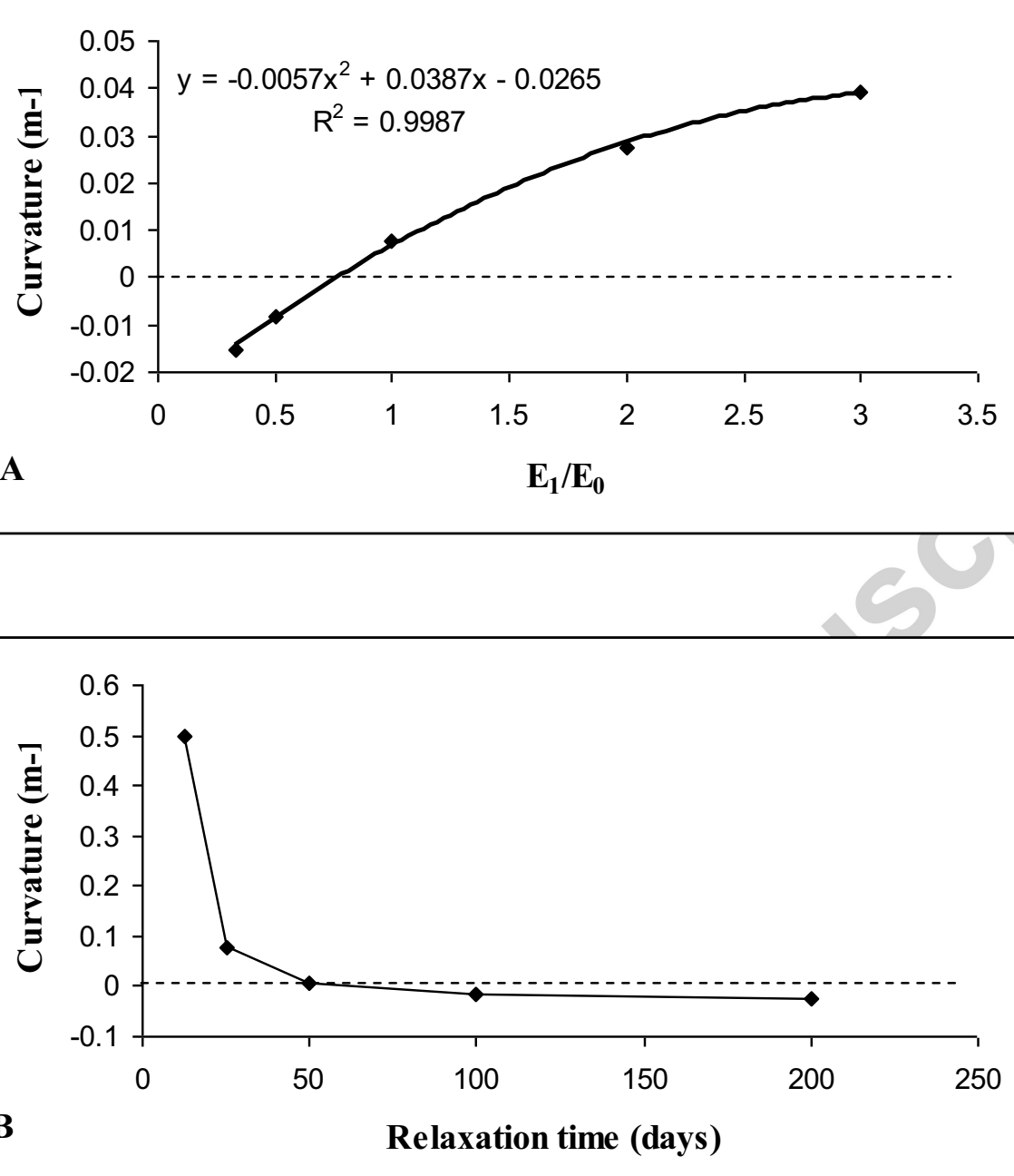

Figure 10 

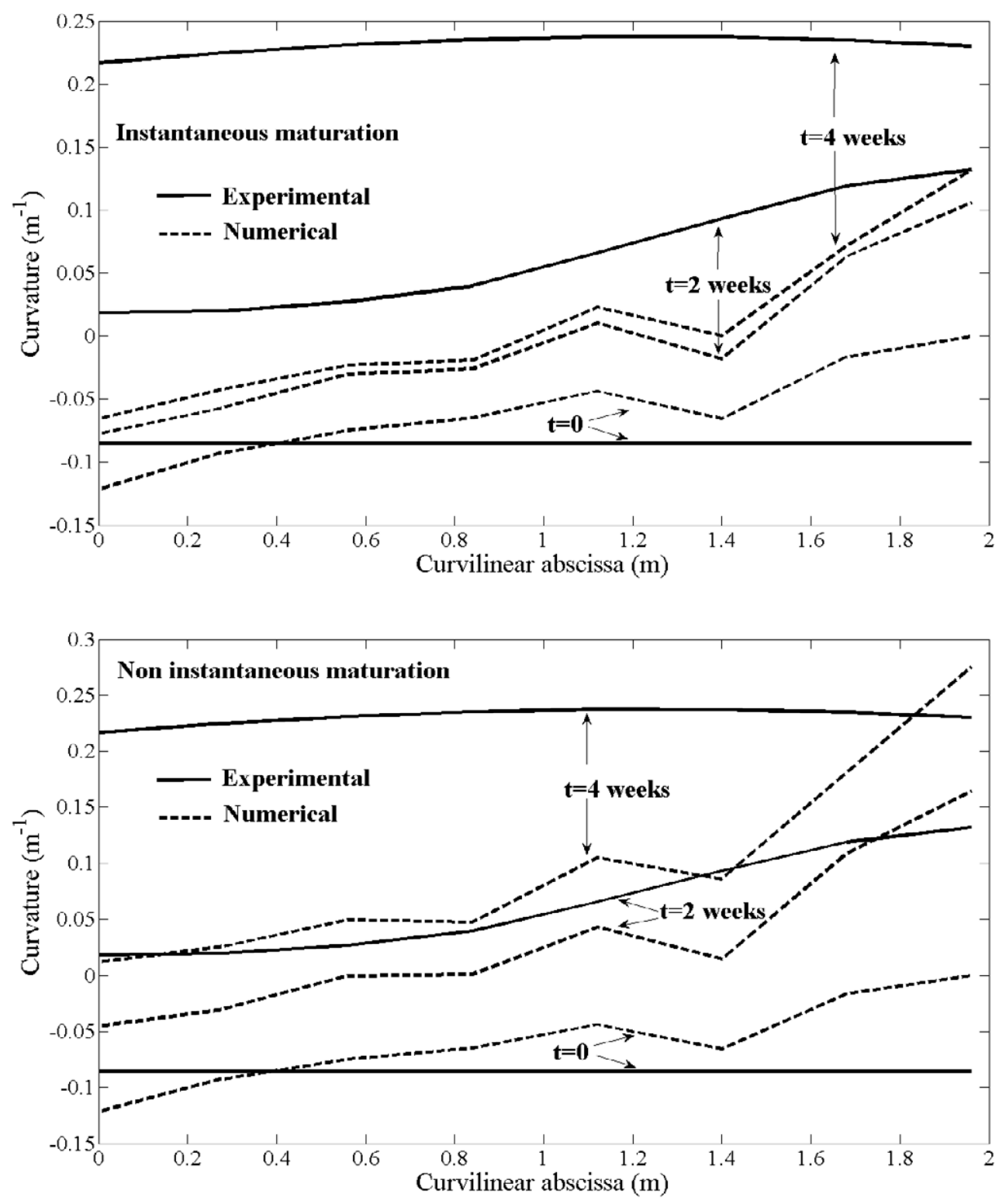

Figure 11 

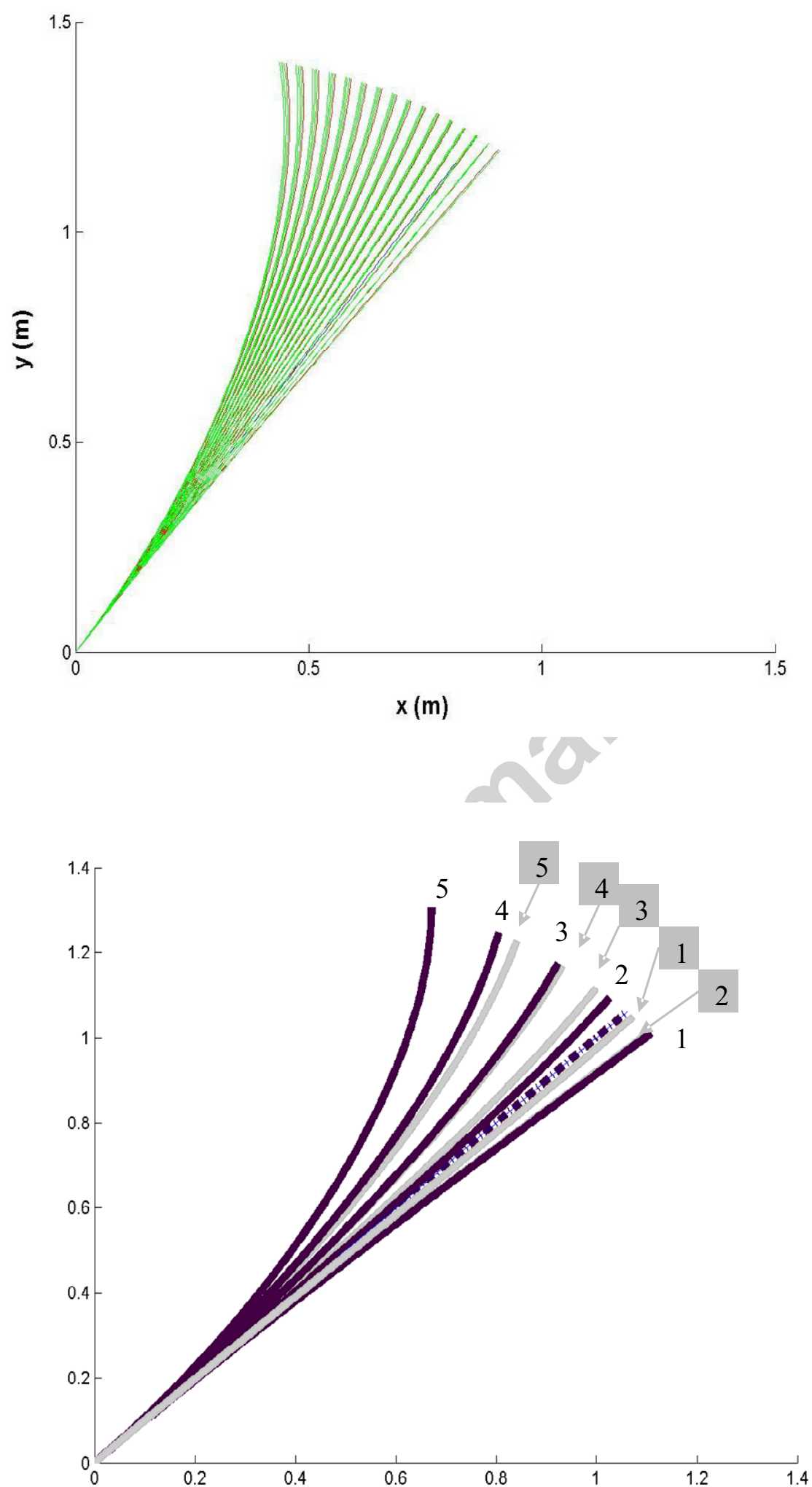

Figure 12 


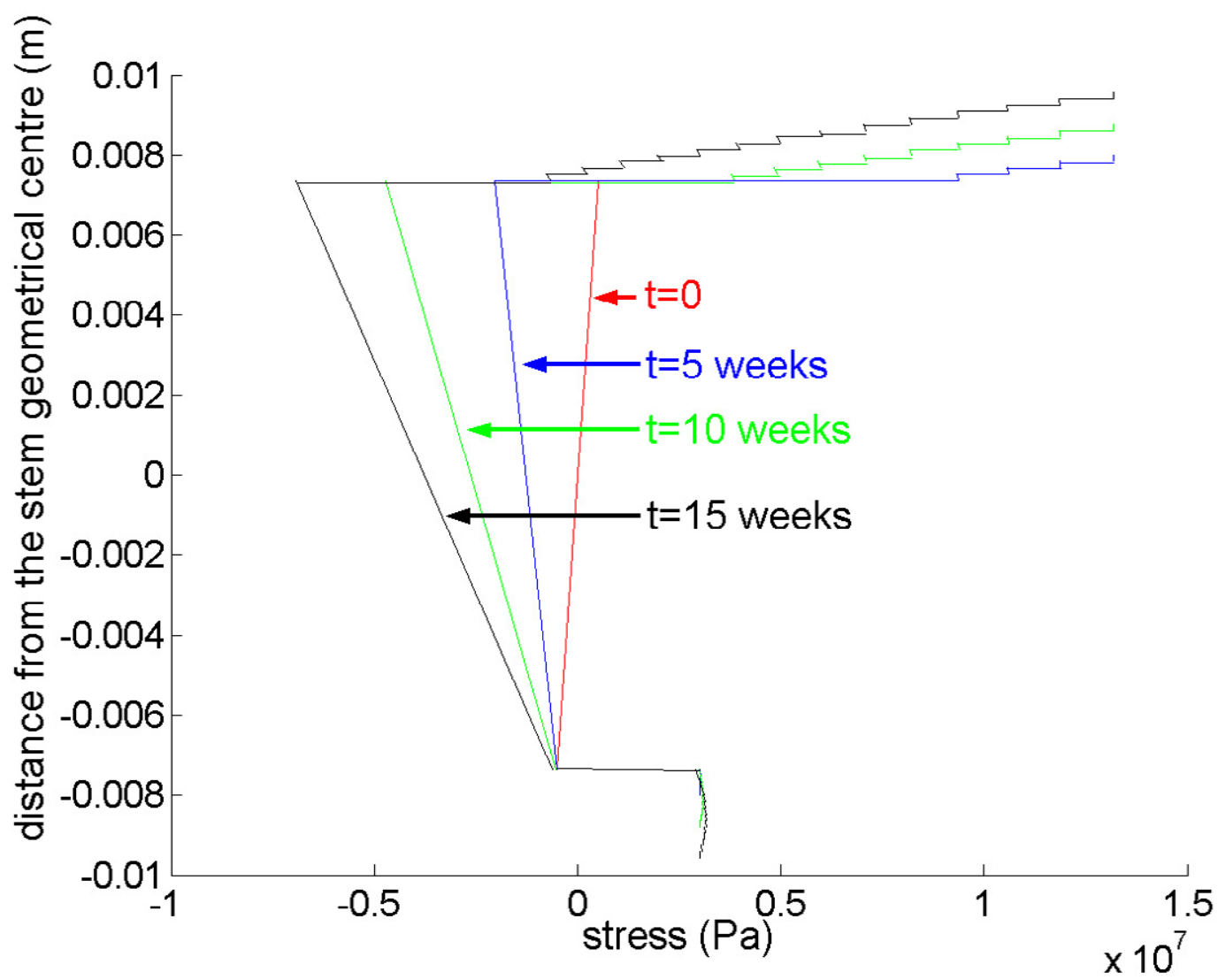

Figure 13 


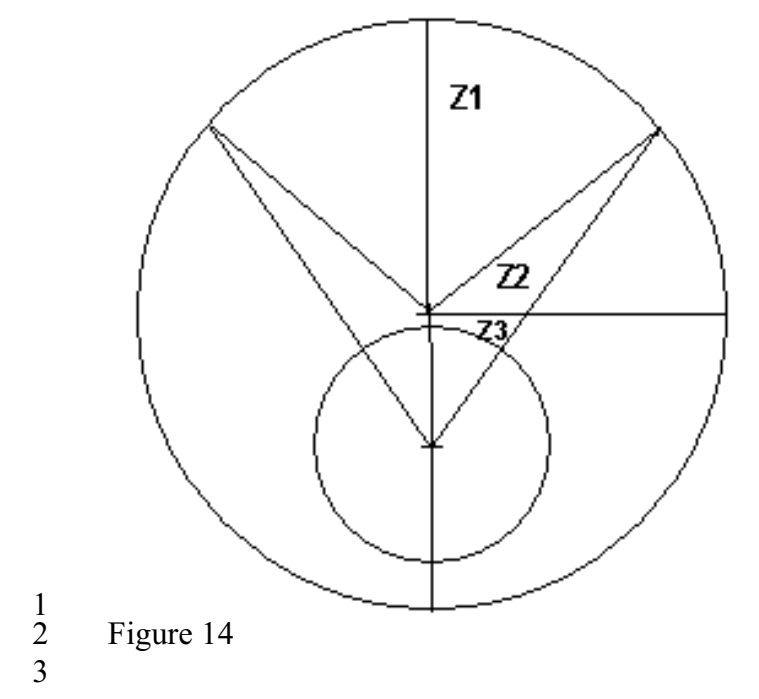

